September $2000 \quad$ • NREL/SR-520-28789

\title{
Post-Lamination Manufacturing Process Automation for Photovoltaic Modules
}

\section{Annual Technical Progress Report 15 June 1999-14 July 2000}

M.J. Nowlan, J.M. Murach, S.F. Sutherland,

E.R. Lewis, and S.J. Hogan

Spire Corporation

Bedford, Massachusetts
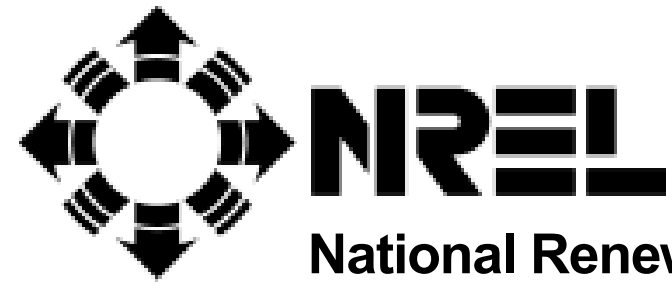

National Renewable Energy Laboratory

1617 Cole Boulevard

Golden, Colorado 80401-3393

NREL is a U.S. Department of Energy Laboratory

Operated by Midwest Research Institute $\bullet$ Battelle $\bullet$ Bechtel

Contract No. DE-AC36-99-G010337 


\section{Post-Lamination Manufacturing Process Automation for Photovoltaic Modules}

\section{Annual Technical Progress Report 15 June 1999-14 July 2000}

M.J. Nowlan, J.M. Murach, S.F. Sutherland, E.R. Lewis, and S.J. Hogan Spire Corporation Bedford, Massachusetts

NREL Technical Monitor: M. Symko-Davies

Prepared under Subcontract No. ZAX-8-17647-04

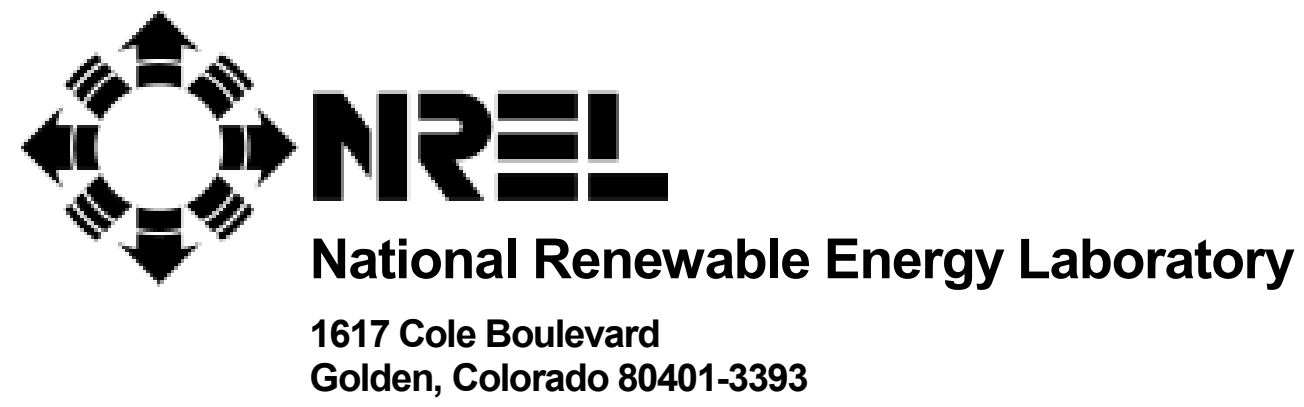

NREL is a U.S. Department of Energy Laboratory

Operated by Midwest Research Institute $\bullet$ Battelle $\bullet$ Bechtel

Contract No. DE-AC36-99-G010337 


\section{NOTICE}

This report was prepared as an account of work sponsored by an agency of the United States government. Neither the United States government nor any agency thereof, nor any of their employees, makes any warranty, express or implied, or assumes any legal liability or responsibility for the accuracy, completeness, or usefulness of any information, apparatus, product, or process disclosed, or represents that its use would not infringe privately owned rights. Reference herein to any specific commercial product, process, or service by trade name, trademark, manufacturer, or otherwise does not necessarily constitute or imply its endorsement, recommendation, or favoring by the United States government or any agency thereof. The views and opinions of authors expressed herein do not necessarily state or reflect those of the United States government or any agency thereof.

Available electronically at http://www.doe.gov/bridge

Available for a processing fee to U.S. Department of Energy

and its contractors, in paper, from:

U.S. Department of Energy

Office of Scientific and Technical Information

P.O. Box 62

Oak Ridge, TN 37831-0062

phone: 865.576.8401

fax: 865.576.5728

email: reports@adonis.osti.gov

Available for sale to the public, in paper, from:

U.S. Department of Commerce

National Technical Information Service

5285 Port Royal Road

Springfield, VA 22161

phone: 800.553.6847

fax: 703.605.6900

email: orders@ntis.fedworld.gov

online ordering: http://www.ntis.gov/ordering.htm

Printed on paper containing at least $50 \%$ wastepaper, including $20 \%$ postconsumer waste 


\section{TABLE OF CONTENTS}

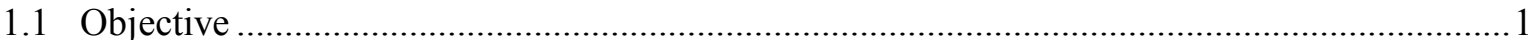

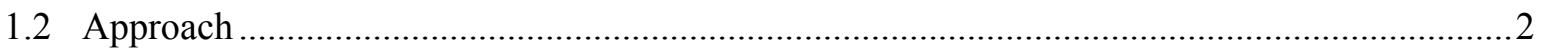

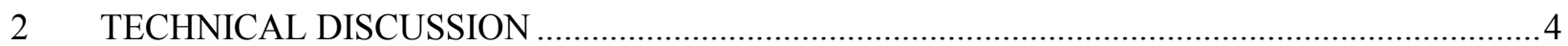

2.1 Task 5 - Design Integrated Edge Process System .............................................................. 4

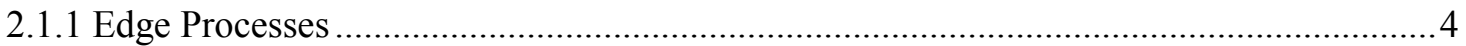

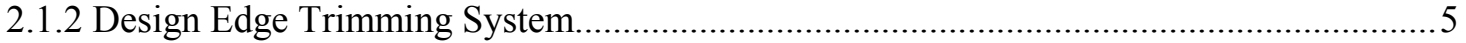

2.1.3 Design Edge Sealing and Framing System ................................................................. 14

2.2 Task 6 - Initial Fabrication of Edge Process System ..........................................................29

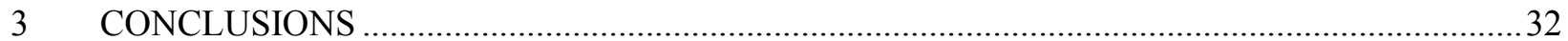

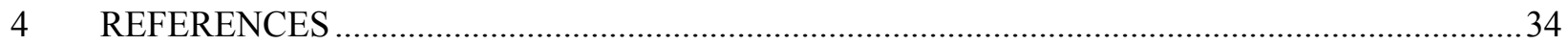




\section{LIST OF ILLUSTRATIONS}

1 Typical manual process sequence for post-lamination module manufacturing. ................................. 1

2 Process sequence for automated post-lamination module manufacturing...........................................2

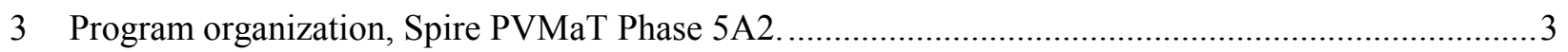

4 Module edge cross-sections after lamination, trimming, sealing, and framing................................. 4

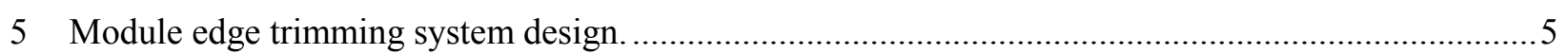

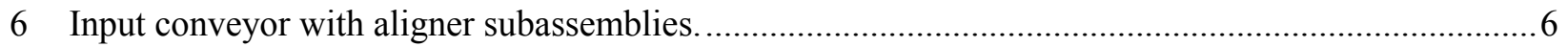

7 Module lift and belt conveyor assemblies, module edge trimmer..................................................... 7

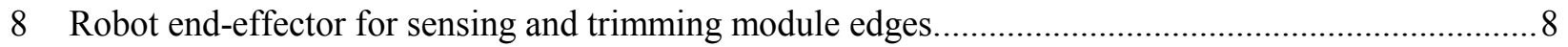

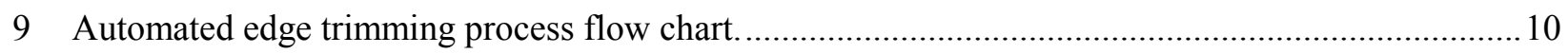

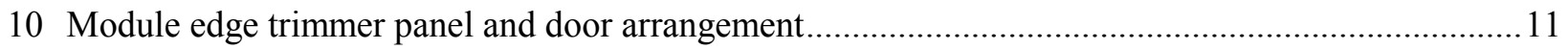

11 Trimmer control system block diagram..................................................................................... 12

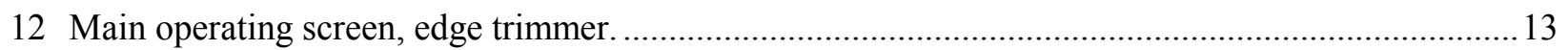

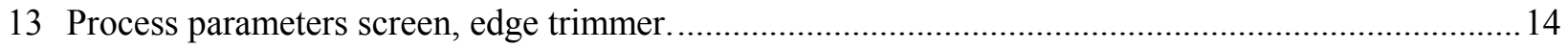

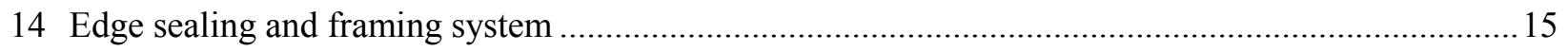

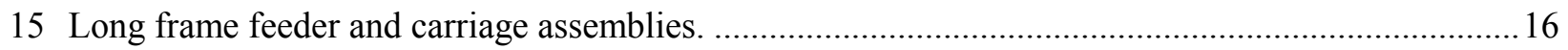

16 Side view of the frame feeder, shown loaded with 60 frame members............................................. 17

17 Three-axis pneumatic pick-and-place mechanism for long frame members. .................................... 18

18 Vacuum hand assembly for long and short frame member pick-and-place assemblies. .....................18

19 Short frame feeder, key press, and carriage assemblies. .............................................................. 19

20 Four-axis pneumatic pick-and-place mechanism for short frame members.....................................20

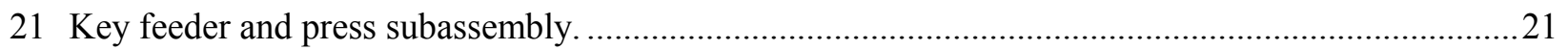

22 Short frame carriage assembly with two frame members ready for pick-up...................................22

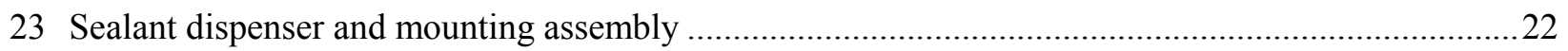

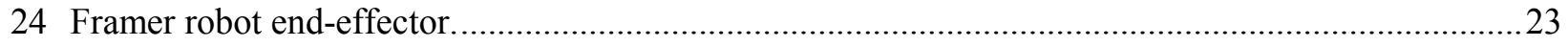

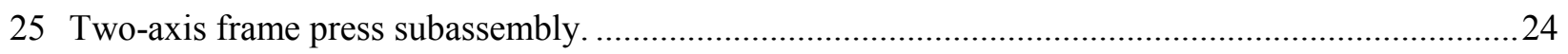

26 Top-level process flow chart for the module framing system. ......................................................26

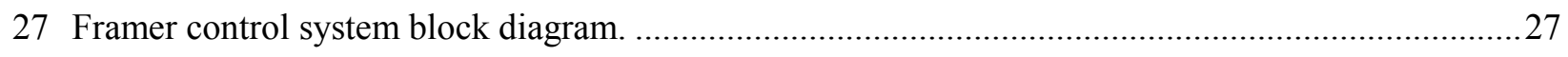

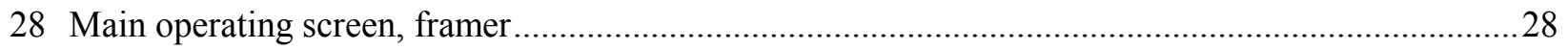

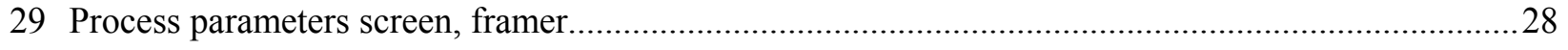

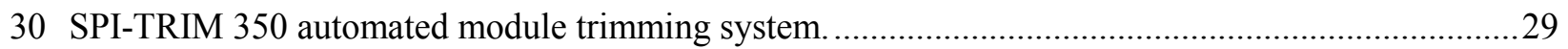

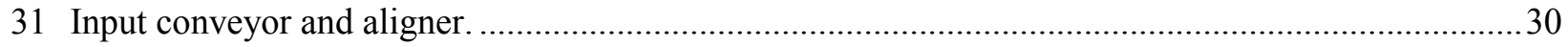

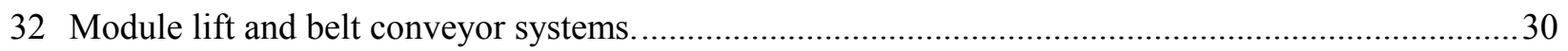

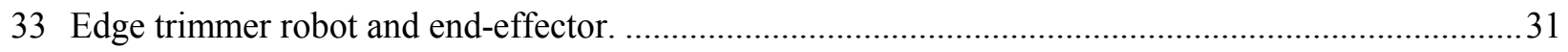

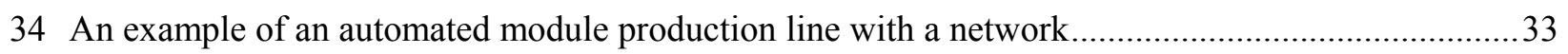




\section{LIST OF TABLES}

Page

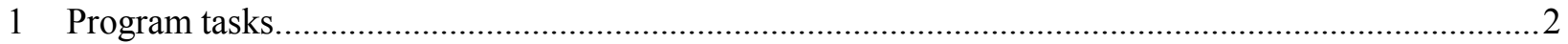

2 Schedule for demonstrating new module process automation. …..................................................... 3

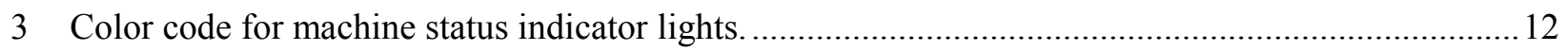




\section{$1 \quad$ INTRODUCTION}

This is Spire Corporation's Annual Technical Progress Report for Phase 2 of a program entitled "Post-Lamination Manufacturing Process Automation For Photovoltaic Modules." This program was made possible by cost-share funding from the U. S. Department of Energy under National Renewable Energy Laboratory (NREL) subcontract No. ZAX-8-17647-04. The Phase 2 period was 13 months, from June 15, 1999 to July 14, 2000.

This program is part of Phase 5A2 of the Photovoltaic Manufacturing Technology (PVMaT) project. The Technical Monitoring Team members are Dr. Martha Symko-Davies (NREL), Mr. Michael Quintana (Sandia National Laboratories), and Mr. Steve Rummel (NREL).

\subsection{Objective}

Spire is addressing the PVMaT project goals of photovoltaic (PV) module cost reduction and improved module manufacturing process technology. New cost-effective automation processes are being developed for post-lamination PV module assembly, where post-lamination is defined as the processes after the solar cells are encapsulated. These processes apply to both crystalline and thin film solar cell modules. Four main process areas are being addressed:

- module buffer storage and handling between steps

- module edge trimming, edge sealing, and framing

- junction box installation

- testing for module performance, electrical isolation, and ground path continuity

Currently, little or no automation is used by PV module manufacturers for these post-lamination processes. A typical manual process sequence is shown in Figure 1. The development and implementation of automated systems are expected to result in significant labor cost savings, improved product quality, and increased throughput. A reduction in the occurrence of repetitive stress injuries may also be achieved by eliminating product lifting and manual edge trimming tasks.

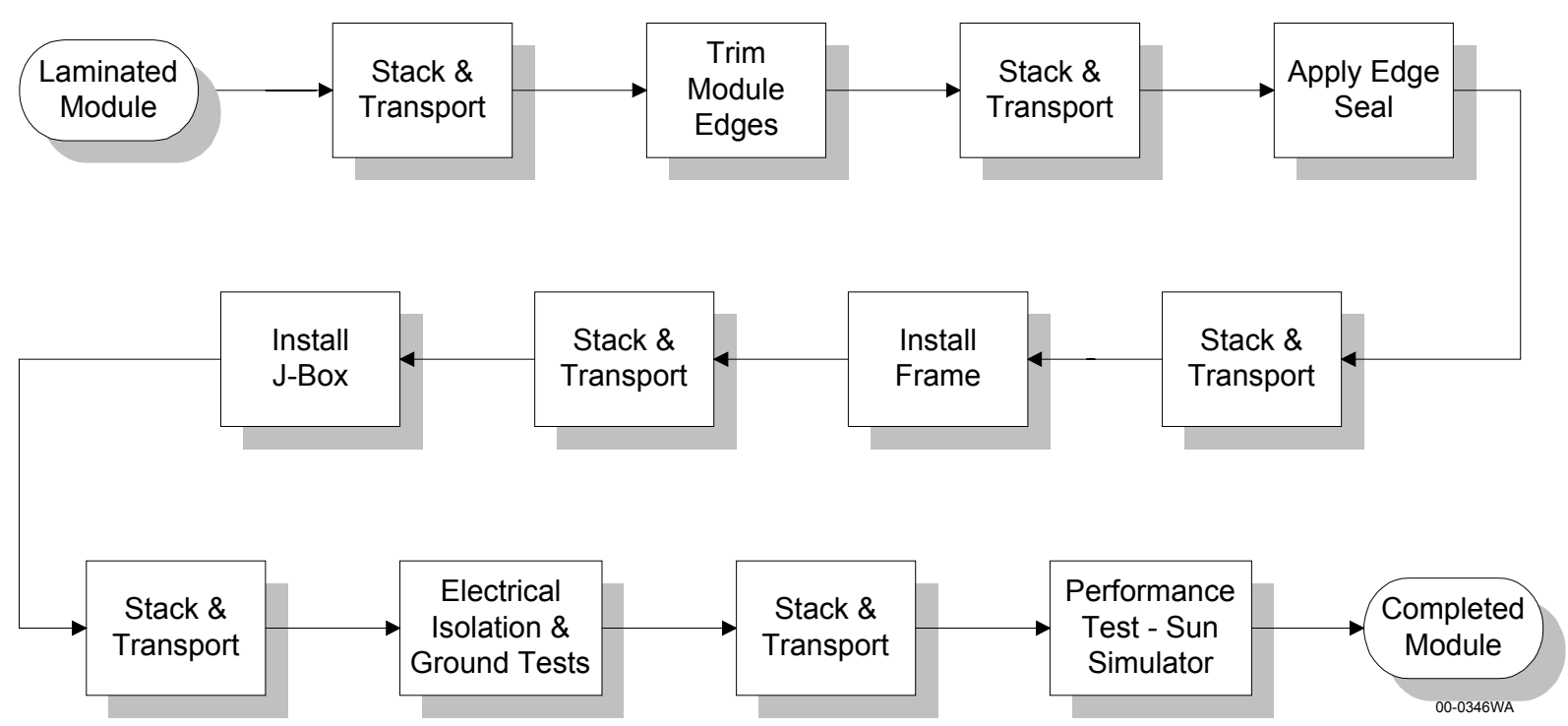

Figure 1 Typical manual process sequence for post-lamination module manufacturing. 


\subsection{Approach}

A three year, three phase program is underway for developing and demonstrating new automated systems for post-lamination PV module manufacturing processes. The systems are (1) a module buffer storage system, including conveyor loading/unloading and module storage, (2) an integrated edge processing system, with automated edge trimming, edge sealing, and framing capabilities, (3) a junction box installation system, and (4) an integrated module testing system that combines electrical isolation testing, ground continuity testing, and module performance testing. These systems are being designed to be combined to form an integrated production line, as shown in Figure 2.

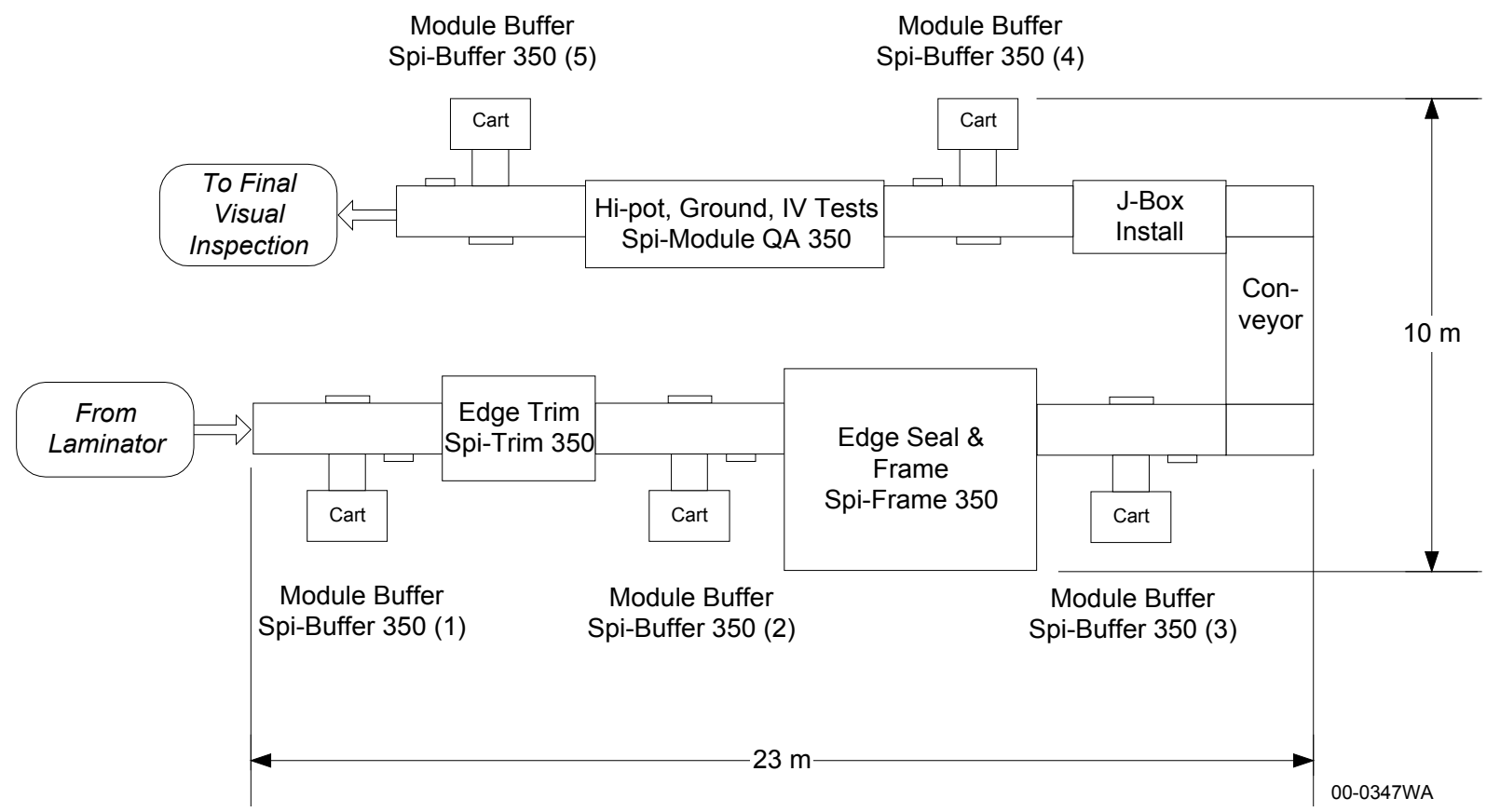

Figure 2 Process sequence for automated post-lamination module manufacturing; plan view.

Program tasks are listed in Table 1. Tasks 1 through 4 were completed in Phase 1, while Tasks 5 and 6, the design and initial fabrication of an integrated edge process system, form the subject of this report.

Table 1 Program tasks.

\begin{tabular}{|c|c|}
\hline Phase & Task \\
\hline 1 - April 98 to June 99 & $\begin{array}{l}1 \text { - Design Definition } \\
2 \text { - Develop Buffer System } \\
3 \text { - Edge Process Development } \\
4 \text { - Develop Integrated Test System }\end{array}$ \\
\hline 2 - June 99 to July 00 & $\begin{array}{l}5 \text { - Design Integrated Edge Process System } \\
6 \text { - Initial Fabrication of Integrated Edge Process System }\end{array}$ \\
\hline 3 - July 00 to July 01 & $\begin{array}{l}7 \text { - Fabricate and Integrate Edge Framer System } \\
8 \text { - Junction Box Process Development } \\
9 \text { - Develop Junction Box Installation System }\end{array}$ \\
\hline
\end{tabular}


As the prototype automation systems are developed, they are being evaluated with module components from several module manufacturers. The schedule for demonstrating these systems is provided in Table 2.

Table 2 Schedule for demonstrating new module process automation.

\begin{tabular}{|c|c|}
\hline Automated Process & Schedule \\
\hline $\begin{array}{l}\text { - } \text { Module buffer storage with conveyor load/unload } \\
\text { - } \quad \text { Trim module edges } \\
\text { - } \text { Apply edge seal and install frame } \\
\text { - Install junction box, module leads, and diodes } \\
\text { - Transport, probe, and test modules for electrical } \\
\text { isolation, ground continuity, and performance (I-V } \\
\text { curve) }\end{array}$ & $\begin{array}{l}\text { Phase 1, June } 1999 \text { (completed) } \\
\text { Phase 2, July } 2000 \\
\text { Phase 3, December } 2000 \\
\text { Phase 3, July } 2001 \\
\text { Phase 1, June } 1999 \text { (completed) }\end{array}$ \\
\hline
\end{tabular}

Spire assembled a team for implementing this program that includes several major US module producers and the Automation \& Robotics Research Institute (ARRI) at the University of Texas at Arlington (UTA). Program team members and their responsibilities are outlined in Figure 3.

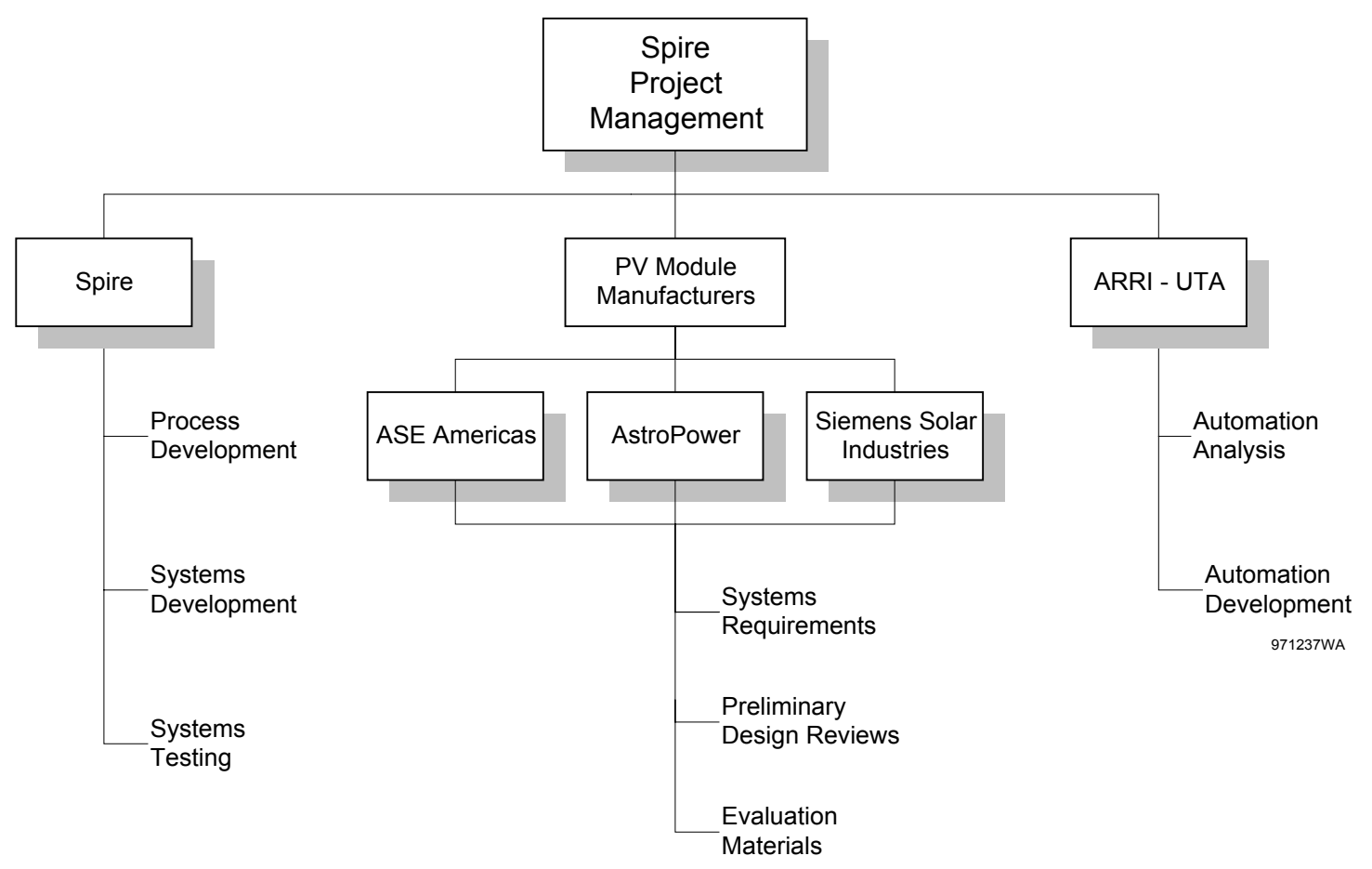

Figure 3 Program organization, Spire PVMaT Phase 5A2.

Major US module manufacturers that teamed with Spire in this effort include ASE Americas, Billerica, MA; AstroPower, Inc., Newark, DE; and Siemens Solar Industries, Camarillo, CA. These and other PV manufacturers provided information on their production requirements and feedback on Spire's automated systems designs. They also provided solar cell laminates and other module materials which Spire is using to evaluate the automated processes being developed in the program. 


\section{TECHNICAL DISCUSSION}

\subsection{Task 5 - Design Integrated Edge Process System}

An integrated edge process system for photovoltaic modules was designed in this task. This system consists of two automated machines, an edge trimmer for trimming excess encapsulant and back cover film from module edges after lamination, and an edge sealer and framer for installing edge sealant and frames on trimmed modules. Both machines have conveyors for module transport and use standard handshake protocols, allowing them to be placed together or separately to suit the manufacturer's process sequence.

\subsubsection{Edge Processes}

After module lamination, the laminate edges are trimmed, a sealant is applied to the edges, and a frame is installed. The edge trimming process removes excess encapsulant and back cover film from the module laminate edges and disposes of the excess material. The edge sealing process applies a sealant around the module edges by dispensing a bead of sealant in a channel in each of four module frame sections. The sealant acts as an adhesive between the frame and the module edges and as a gasket to cushion the glass in the aluminum frame. The sealant bead approach was selected over an edge tape method because the sealant material cost is lower and the process is more suitable to automation. The frame sections are attached to the module and joined at the corners with fasteners. An illustration of the edge processes is provided in Figure 4.

1. Module cross-section after lamination

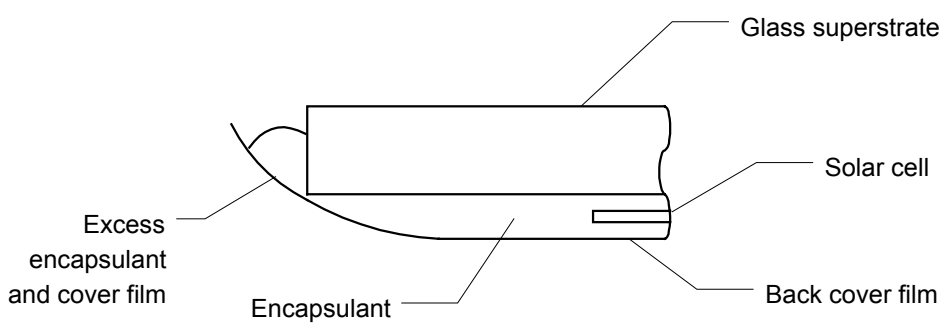

2. Edge trimmed

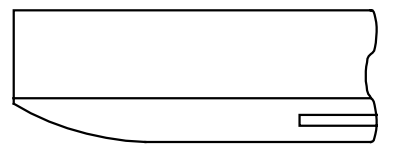

3. Frame installed with edge sealant in channel

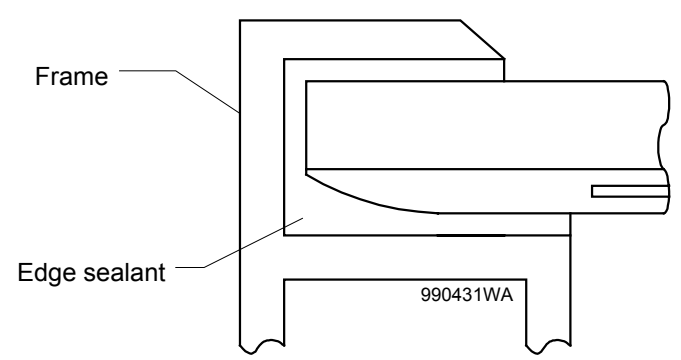

Figure 4 Module edge cross-sections after lamination, trimming, sealing, and framing.

During Phase 1, Spire collaborated with ARRI to develop and demonstrate module edge trimming, edge sealing, and framing processes that were designed for automation. Concepts for implementing these processes in production were defined. These concepts provided the basis for the production prototype automation design and development work done in this phase of the program. 


\subsubsection{Design Edge Trimming System}

A detailed design was developed for a prototype automated production edge trimming system, designated the SPI-TRIM ${ }^{\mathrm{TM}} 350$. The system's main components include a conveyor system, a module aligner, a module lift, a four-axis Cartesian robot, and an end-effector for edge sensing and trimming. The design is shown in Figure 5, with panels and doors removed to show the system's functional elements.

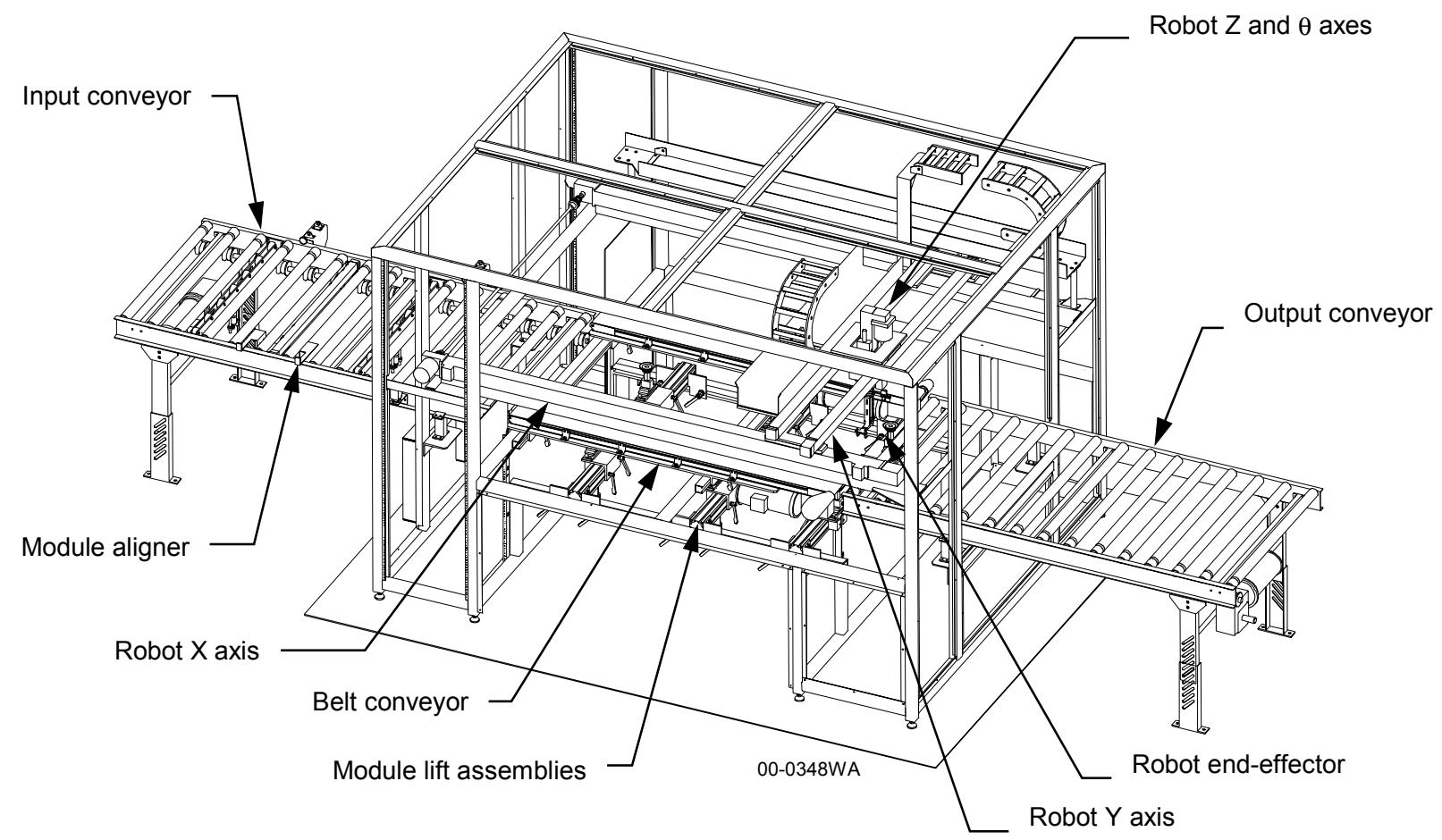

Figure 5 Module edge trimming system design; panels and doors not shown.

The conveyor system has three sections: input, trimming, and output. Motor driven rollers transport modules on the input and output conveyors. The rollers are covered with urethane to provide traction and prevent damage to modules with glass surfaces. A pair of motor-driven belt conveyors are used to transport modules in the center trimming section. The belt conveyors provide clearance for positioning the module lift assemblies, while eliminating the potential for stalling which could occur if rollers were used, since trimmed material from modules could wrap around the rollers.

The module aligner, built into the input conveyor, provides coarse alignment for untrimmed modules. The aligner consists of two cross-roller assemblies, two retractable stops, an alignment arm, and optical sensors, as shown in Figure 6.

When the trimmer input conveyor is available and the upstream process signals that it is ready to send a module, the input conveyor turns on to transport the module into the aligner section of the machine. The conveyor turns off when the leading edge of the module breaks an infrared beam. The module is lifted up above the conveyor rollers by the cross-roller assemblies, which contain sets of wheels that are perpendicular to the roller direction. An arm driven by an air cylinder gently pushes the module against a pair of stops on the opposite edge of the conveyor. The module is now aligned with one edge against the module stops. 


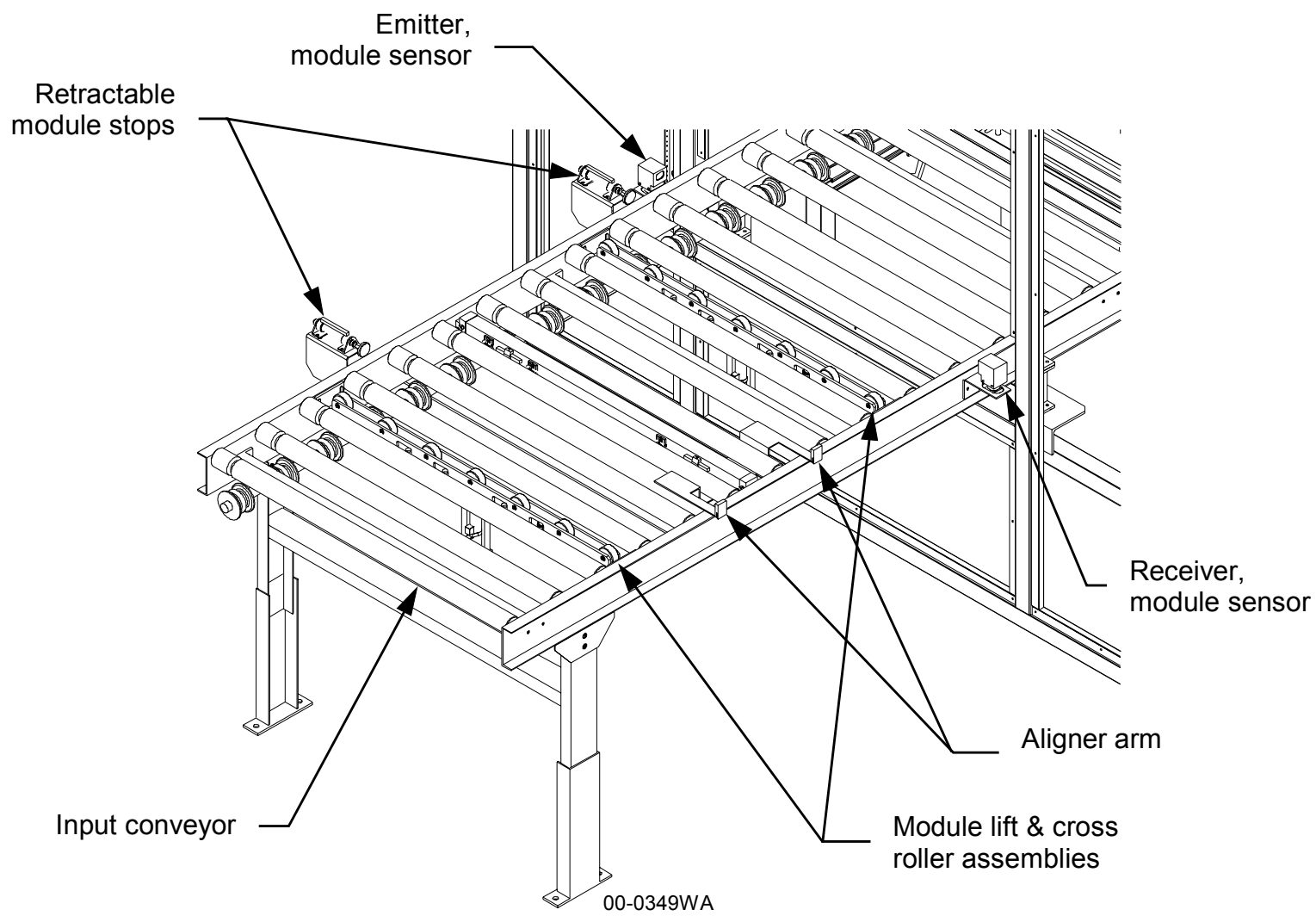

Figure 6 Input conveyor with aligner subassemblies.

An optical sensor detects when the module reaches the module stops, which signals the two cross roller assemblies to retract, placing the module back on the conveyor rollers. First the aligner arm and then the module stops retract away from the module. The module is now ready to enter the trimming area. In production, the aligned module typically will wait on the input conveyor until the trimming process is completed on the previous module, since the alignment process is faster than the trimming process.

The aligner assemblies are similar in design to those developed for the module buffer and the module tester, developed in Phase 1 of this program, with two improvements:

- An optical emitter and receiver are mounted on opposite sides of the input conveyor, in place of a retractable mechanical stop. When a module travelling down the conveyor breaks the infrared beam, the conveyor turns off to stop the module at the aligner. This approach can be used here because alignment in the conveyor travel direction is not critical at this point in the process. The aligner is simplified by eliminating the stop mechanism.

- Two retractable stops are used in place of fixed stops to oppose the action of the alignment arm. These stops allow a module to be aligned even if its edge is as much as $50 \mathrm{~mm}(2.0$ ") beyond the edge of the conveyor rollers. This feature was added to align modules that do not track perfectly on the conveyor system from an upstream station.

After alignment, the module is transported into the trimming section on two belt conveyors, as shown in Figure 7. Six large rubber vacuum cups mounted on guided air cylinders grip the module 
securely and lift it up above the belt surface for trimming. These lift assemblies are mounted on rails with quick release clamps that allow the lift locations to be adjusted in both $\mathrm{x}$ and $\mathrm{y}$ directions, as required to accommodate various size modules. Similarly, the two conveyor belts are mounted with quick release clamps that allow the belt positions to be adjusted in the direction perpendicular to the belt travel direction, as needed for various width modules.

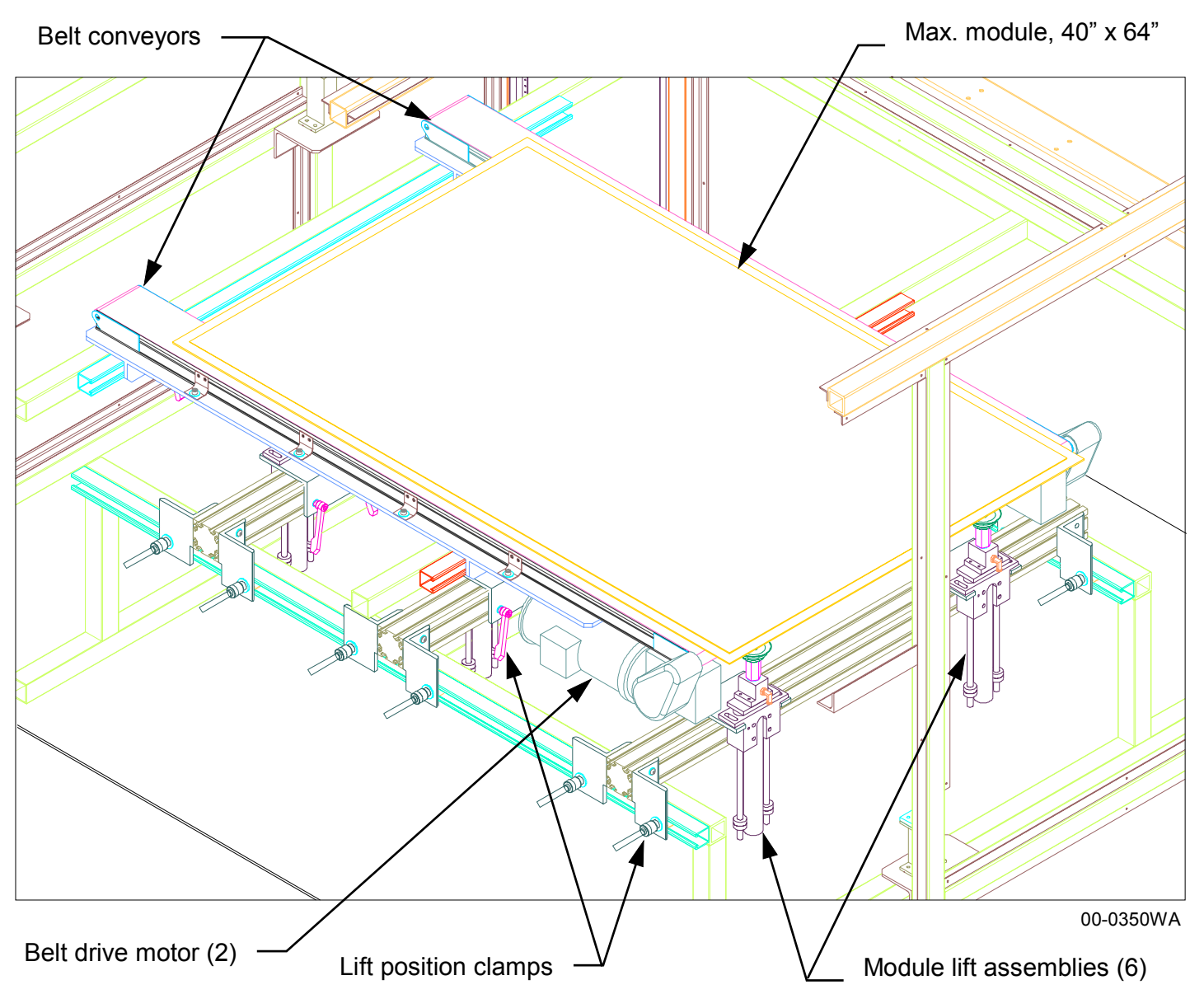

Figure $7 \quad$ Module lift and belt conveyor assemblies, module edge trimmer. (Frame cut away and robot, panels, and doors not shown for clarity.)

Module edge sensing and trimming operations are performed by a four axis $(\mathrm{x}, \mathrm{y}, \mathrm{z}$, and $\theta)$ Cartesian robot. All four axes are servo motor controlled. The horizontal (x and y) axes have belt driven stages with roller bearings. The vertical $(\mathrm{z})$ axis is a linear electric cylinder with a ball screw and guide rods. The rotation $(\theta)$ axis is a $15 \mathrm{~cm}(6$ ") diameter rotary table, attached to the $\mathrm{z}$ axis.

The robot transports an end-effector with photoelectric sensors for detecting module edges and a hot knife for trimming excess material from the module edges. The trimmer end-effector, shown in Figure 8, consists of a hot knife for trimming module encapsulant and back cover material, two fibercoupled optical sensors for finding module glass edges, and two long-range optical sensors for controlling module position in the conveyor travel direction. A mechanical clutch is provided between the end-effector and the robot to protect the robot from damage in the event of a collision. 


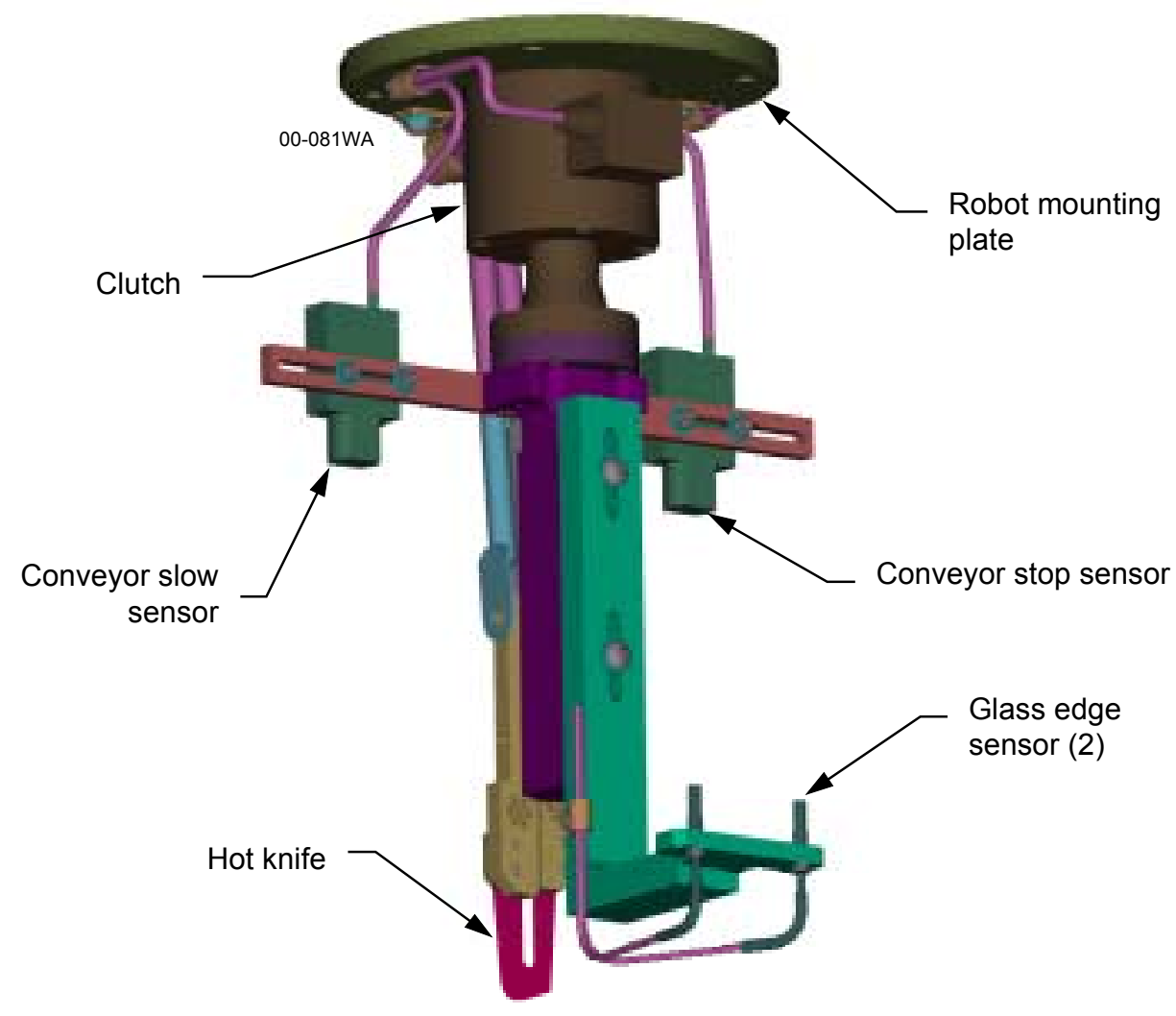

Figure 8 Robot end-effector for sensing and trimming module edges.

The robot positions the long-range sensors over the downstream end of the belt conveyors when waiting for a module to enter the trimming area. When the module reaches the first long-range sensor, the belt conveyor is switched from its normal running speed to slow speed. When the module reaches the second sensor, the belt conveyor is switched off. This two-sensor design allows a reasonably high belt travel speed to meet throughput requirements while providing more accurate module positioning in the belt travel direction than can be obtained with a single sensor.

Once the module is positioned on the belts, the lift system raises the module above the belts and the robot end-effector travels at high speed to each corner of the module. The robot scans the two fiber optic sensors at slow speed across two adjacent glass edges at each corner. Servo position data is captured as each sensor crosses its reflectance threshold. This position data is used to calculate the location of the glass perimeter.

The perimeter data is compared against user-specified tolerances for glass size and angle of each corner. If the glass perimeter fails this dimensional inspection, the specific fault condition is displayed and the module is passed out of the system without being trimmed. If the glass perimeter passes the dimensional inspection, and if the hot knife is within a specified temperature range, the robot trims the four sides of the module with the hot knife. The robot can be programmed to trim at a specified distance (typically $0.5 \mathrm{~mm}$ ) from the glass edge, which greatly extends blade life, given the highly abrasive nature of glass. The robot can also move the knife blade vertically in a periodic motion while trimming, which also extends blade life by varying the spot on the blade which contacts the module. 
After the trimming process is complete, the belt conveyors run at high speed to remove any material that may fall on them during trimming. The lift vacuum is then turned off and the module lift moves down to place the module on the conveyor. If the output roller conveyor is empty, the belt conveyors and the output roller conveyor turn on to transport the module from the trimming station to the output conveyor to clear the trimming area for the next module. When the downstream process signals that it is ready to receive a module, the output conveyor turns on to transport the module out of the trimmer.

A detailed process sequence and a flow chart for edge trimming were written. These documents were used as the basis for specifying sensors and controls and for developing the operating software. The flow chart is shown in Figure 9. A summary of the process sequence follows:

1. Transport a module into the trimmer on a roller conveyor and do a coarse alignment.

2. Transport the module onto a pair of belt conveyors and stop at the trim position.

3. Grip and raise the module up above the belt conveyors with a vacuum lift.

4. Sense the glass edges with a pair of optical sensors and calculate the location of the glass perimeter.

5. Trim the module edges with a hot knife.

6. Empty the belts of cut material.

7. Lower the module onto the belts.

8. Transport the module out of the trimming area.

Three important robot end-effector enhancements were made compared to the system used for process development in Phase 1. ${ }^{1}$ Firstly, an additional fiber optic sensor was added to reduce the sensing time. With two sensors, the end effector can detect two edge points on adjacent sides of a module in one scan, by scanning each corner at a $45^{\circ}$ angle with respect to the module sides. This reduces the number of slow-speed edge scans from eight to four, while still detecting eight edge points.

Secondly, the two sensors are mounted on the opposite side of the robot's rotation $(\theta)$ axis from the hot knife. This design change prevents trimmed material from falling on top of the sensors.

Thirdly, a mechanical clutch was added between the end-effector and the robot. Two methods were considered for protecting the robot arm and its end-effector from damage in the event of a collision: force sensors and a mechanical clutch. Protection must be provided in at least two dimensions, the horizontal plane, and preferably in the vertical dimension as well. After a review of available components, the mechanical clutch approach was selected because it is much simpler to implement in three dimensions in terms of controls and software, while providing mechanical compliance independent of the control system.

The clutch is mounted between the robot arm and the end-effector, and holds the end-effector rigidly in position under normal conditions. In the event that a torque threshold is exceeded in the $x, y$, or $\mathrm{z}$ direction, the bottom part of the clutch pivots away from the top part and a limit switch opens. The limit switch is connected to the robot motor controller, which immediately stops the robot's motion in all four axes. The bottom part of the clutch remains captive in the top part so the end effector remains attached to the robot arm. 


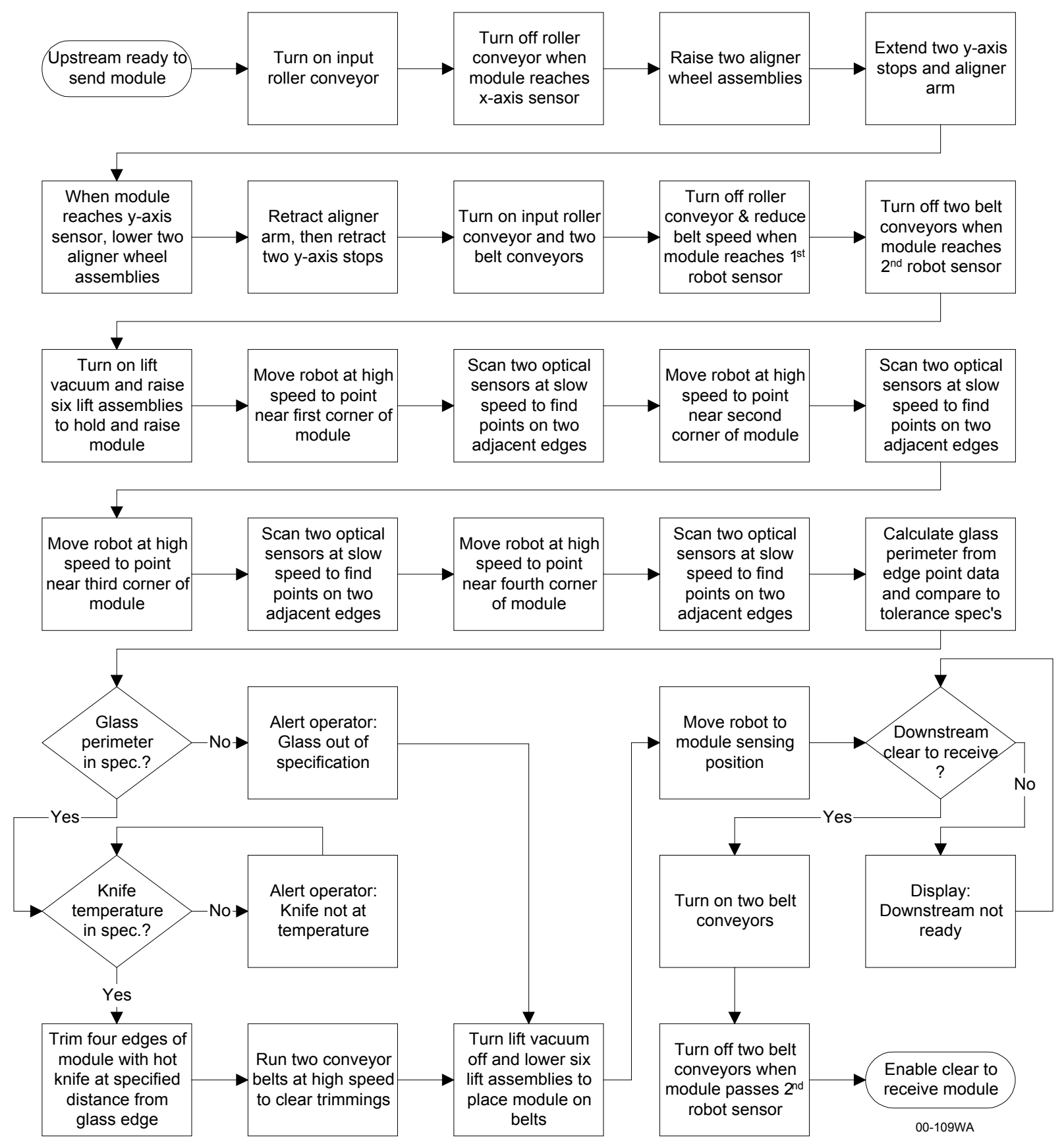

Figure 9 Automated edge trimming process flow chart.

While the clutch provides protection for the robot and its end-effector, it is not sufficient to insure the safety of personnel from possible injury from the hot knife or the moving robot arm, which present cutting and crushing hazards. Light curtains and mechanical guards were considered for operator protection. While either approach can provide a high level of safety, the use of mechanical guards was selected because it reduces the chance of accidentally interrupting machine operation, and it results in a smaller machine footprint. Transparent acrylic panels are used in key locations to allow the machine to be observed during operation. 
An isometric view of the trimmer with its panels in place is shown in Figure 10. Transparent acrylic top panels allow ambient room light into the machine. Hinged doors below the conveyor allow trimmed scrap material to be cleaned out periodically. Clear acrylic sliding doors are provided above the conveyor to allow the machine to be observed during operation and to provide access for set-up or maintenance. All of the doors are electrically interlocked for safety, and emergency stop buttons are installed at each corner of the machine.

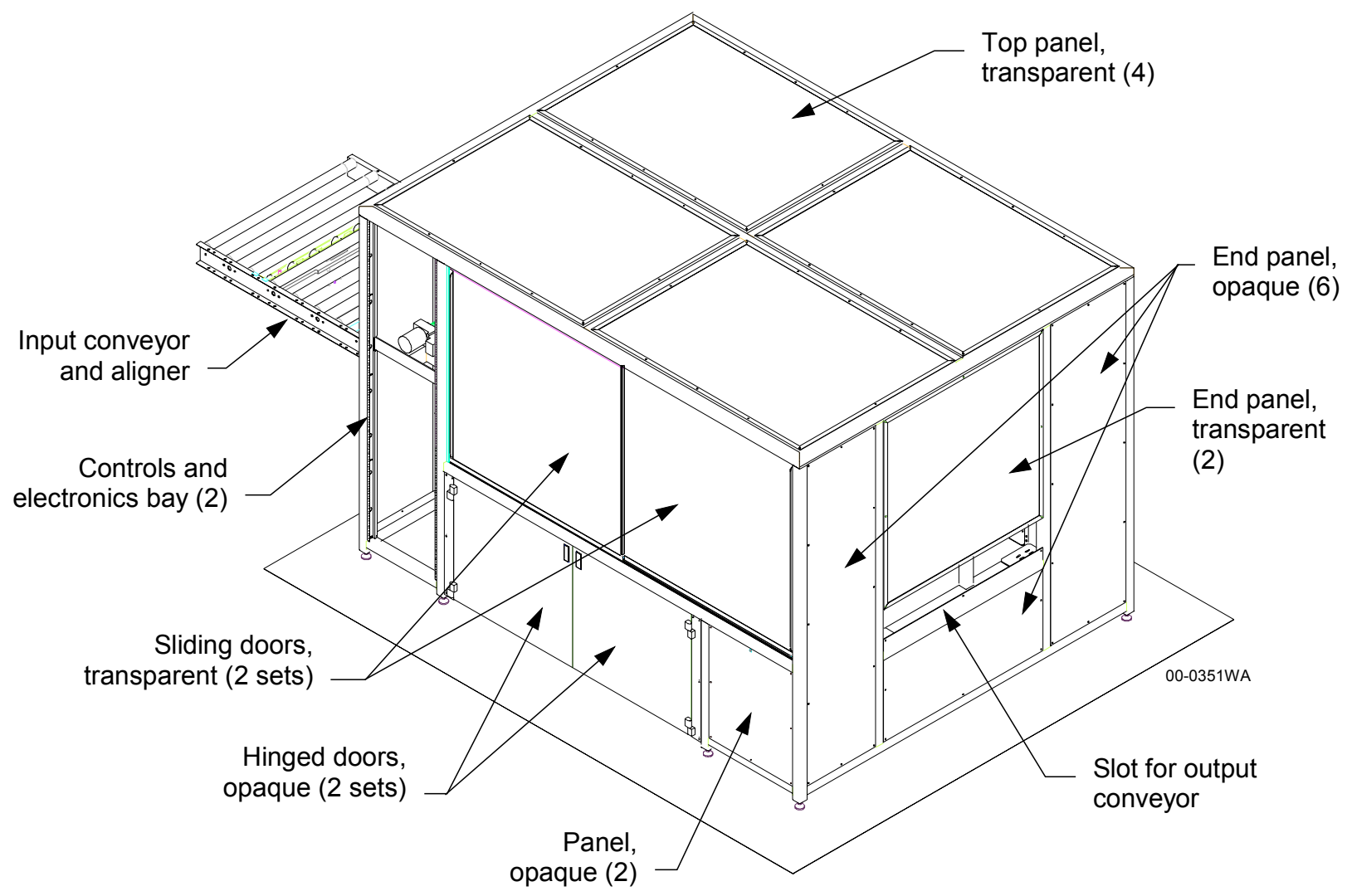

Figure $10 \quad$ Module edge trimmer panel and door arrangement (output conveyor and control panel not shown).

A light tower with red, yellow, and green lights is provided to indicate machine status. The trimmer has six states: run, reset, automatic pause, manual pause, error, and emergency stop. Colors were assigned for each state as listed in Table 3. These color codes are in compliance with the European standard for safety of machinery, EN 60204-1, which is required for machines sold into the member countries of the European Union. ${ }^{2}$ The light tower is mounted at the highest point of the machine, on top of the main frame, for clear visibility in the factory.

Two electronics bays are provided, one in the front and one in the back of the machine. The bays' frames are designed to hold standard 19 inch electronic rack mount equipment. A rack mount PC, a touch screen monitor, and a sliding keyboard shelf are mounted in the front bay for machine control and user interface functions. 
Table 3 Color code for machine status indicator lights.

\begin{tabular}{|l|l|}
\hline \multicolumn{1}{|c|}{ Machine Status } & \multicolumn{1}{c|}{ Color } \\
\hline Emergency Stop & Red \\
Error & Yellow flash \\
Pause, manual & Yellow \\
Pause, automatic & Yellow and green \\
Reset & Green flash \\
Run & Green \\
\hline
\end{tabular}

A control system block diagram for the trimmer is provided in Figure 11. The main controller is an industrial PC running a Microsoft Windows NT operating system. An ethernet connection links the PC with a process controller for servo motor control and handling digital input/output (I/O). A serial (RS232) port connects the PC to a thermocouple converter for monitoring the temperature of the hot knife.

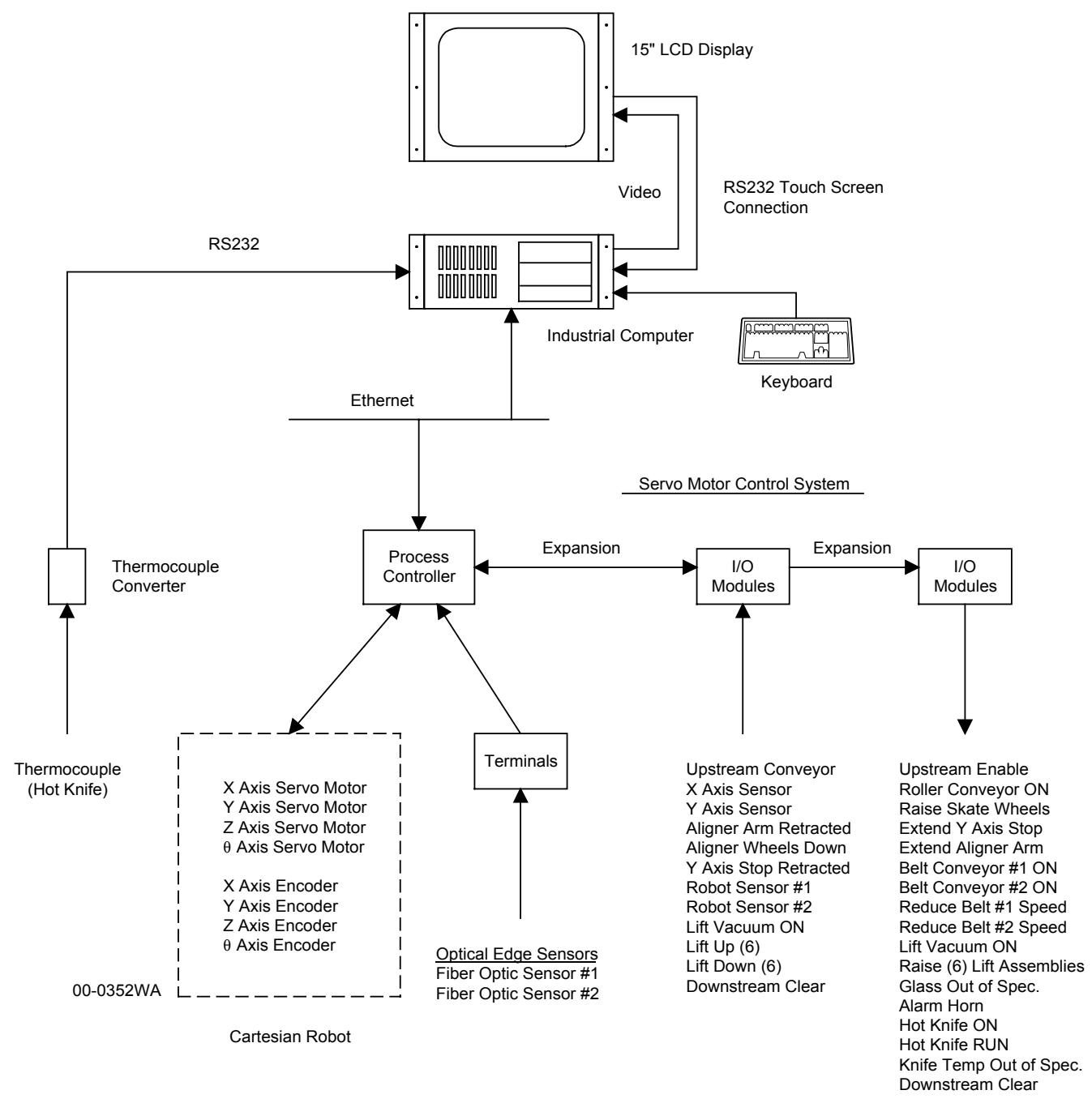

Figure 11 Trimmer control system block diagram. 
An electrical interface standard used by the Surface Mount Equipment Manufacturers Association (SMEMA) was selected to provide a standardized means for the trimmer to communicate with upstream and downstream automation. ${ }^{3}$ The SMEMA interface standard defines a protocol for electrical signals used for transferring product between machines. These signals allow local control, independent of a supervisory controller. This standard is being incorporated into all of the automated systems being developed in this program, allowing processes to be done in the order desired by the manufacturer.

Spire's engineering group created 3-D design models for the mechanical assemblies using Autodesk's Mechanical Desktop solid modeling software. From these models, detail drawings were produced for fabricating parts and mechanical assembly drawings were produced to direct the manufacturing group during assembly. A pneumatic schematic was created to document the compressed air and vacuum systems. Electrical wiring drawings and electrical panel layout drawings were created.

The machine control and user interface software was written in Visual Basic. A touch screen, which is rack mounted in the main frame, provides the main operator interface. The main operating screen is shown in Figure 12. Process parameters are set on a separate screen, shown in Figure 13.

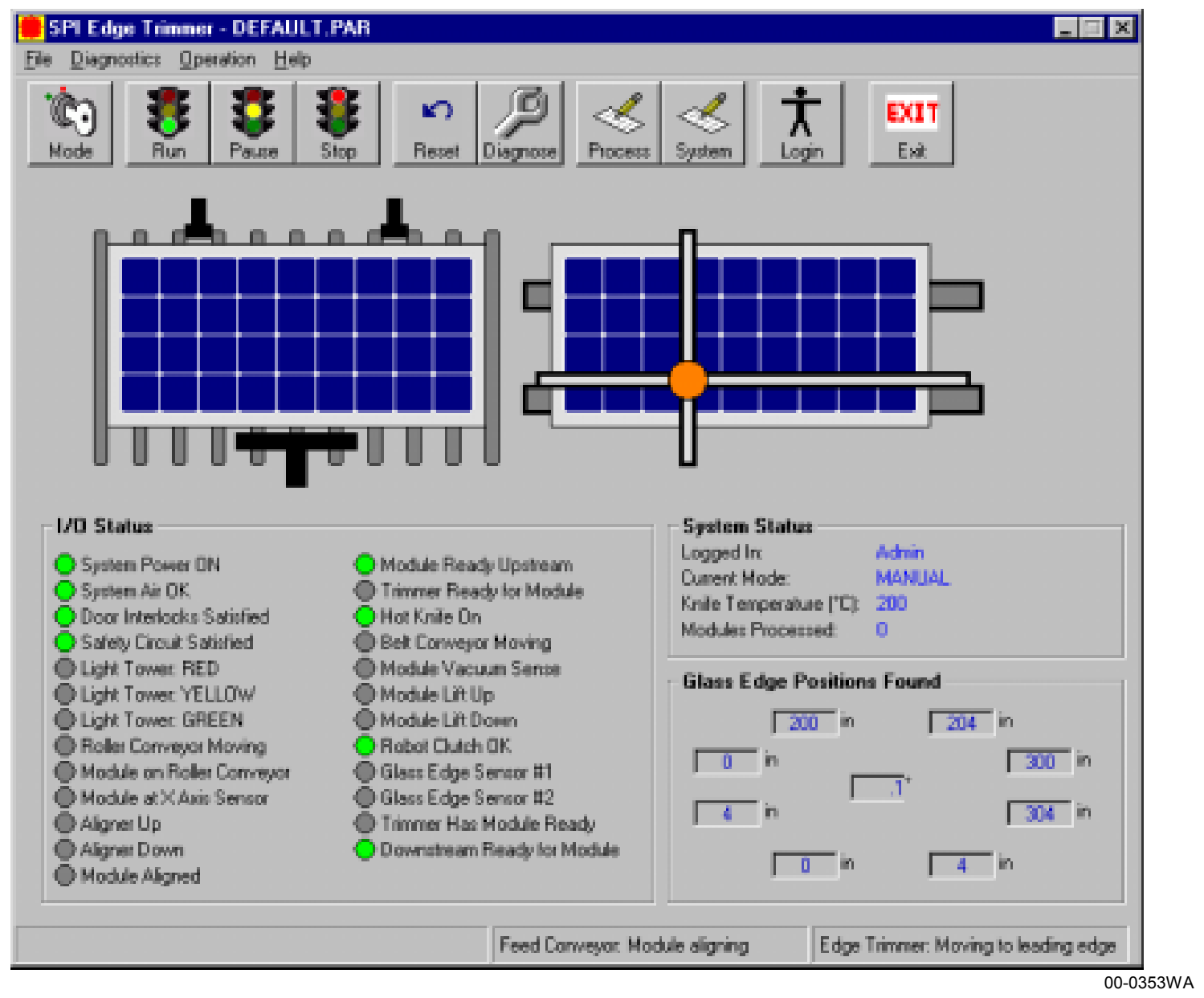

Figure 12 Main operating screen, edge trimmer. 


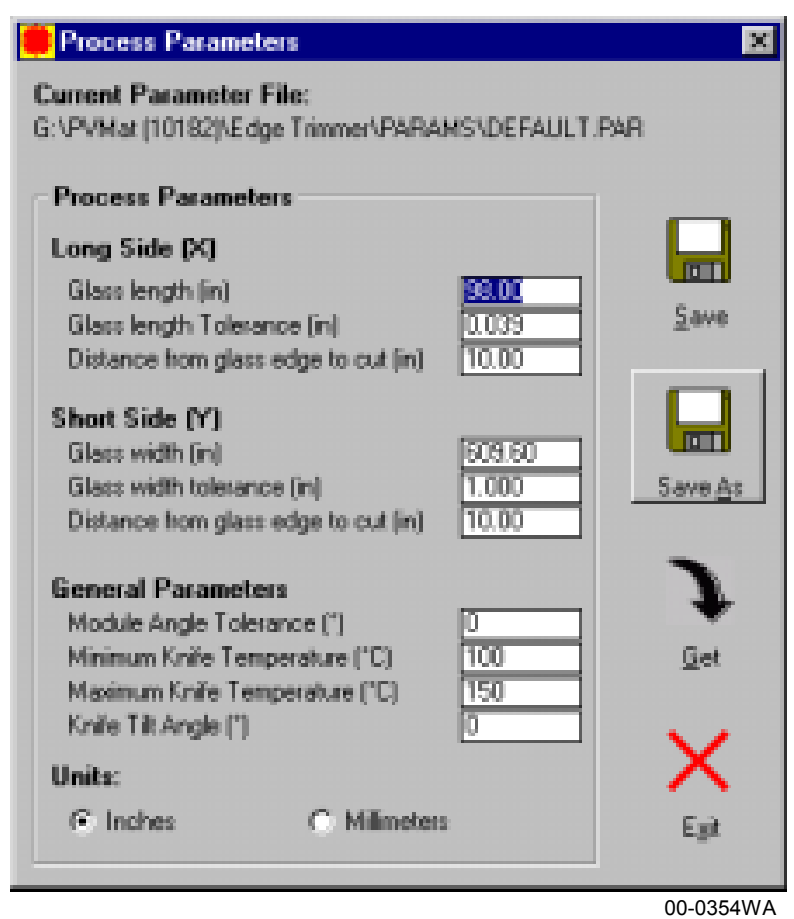

Figure 13 Process parameters screen, edge trimmer.

A system parameters screen is provided to set the Cartesian robot parameters, such as acceleration, velocity, and home positions, for each of four axes. The system parameters are password protected to prevent changes by the operator. Diagnostics screens were also developed to view the status of inputs and outputs. The diagnostics screens allow the outputs to be operated manually (by pressing buttons on the touch screen) to test them for proper operation or to make adjustments for setup or maintenance purposes.

\subsubsection{Design Edge Sealing and Framing System}

A detailed design was developed for a prototype automated production module edge sealing and framing system, designated the SPI-FRAME ${ }^{\mathrm{TM}} 350$. The edge sealer and framer's main components include module transport conveyors, a module aligner, a module lift, a robotic frame transport, a long frame feeder, a short frame feeder, corner key feeders, corner key presses, and a two-axis frame press. The design is shown in Figure 14, with panels and doors removed to show the system's functional elements.

The conveyor system has three sections: input, framing, and output. The conveyors are similar in design to those used in the trimmer. Motor driven rollers transport modules on the input and output conveyors. The rollers are covered with urethane to provide traction and prevent damage to modules with glass surfaces. A pair of motor-driven belt conveyors are used to transport modules in the center framing section. The belt conveyors provide clearance for positioning the module lift assemblies.

When the upstream process signals that it is ready to send a module, the input conveyor turns on and transports the module into the aligner section of the machine. The conveyor turns off when the leading edge of the module breaks an infrared beam. 


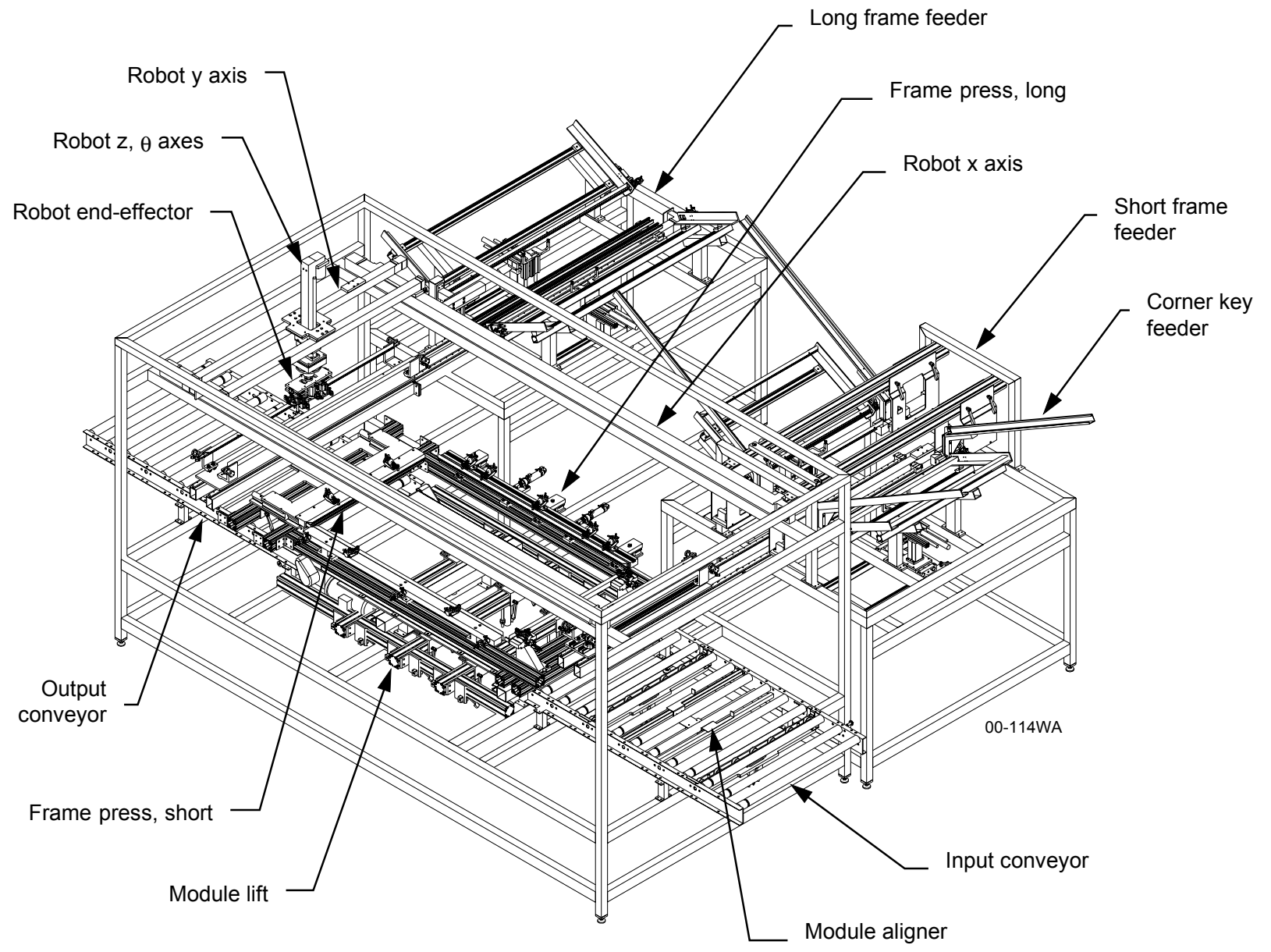

Figure 14 Edge sealing and framing system; panels and doors not shown.

The module aligner is built into the input conveyor. The aligner is similar in design to the aligner developed for the edge trimming system, shown in Figure 6. Modules are lifted up above the conveyor by two sets of wheels that are perpendicular to the roller direction, and an arm driven by an air cylinder gently pushes the module against a pair of stops on the opposite edge of the conveyor.

An optical sensor detects when the module reaches the module stops, which signals the two cross roller assemblies to retract, placing the module back on the conveyor rollers. First the aligner arm and then the module stops retract away from the module. The module is now ready to enter the framing area. In production, the aligned module typically will wait on the input conveyor until the framing process is completed on the previous module, since the alignment process is faster than the framing process.

After alignment, the module is transported into the framing section on two belt conveyors. Seven guided air cylinders, two with large rubber vacuum cups to grip the module and five with plastic pads to support the module, raise up to lift the module above the belt surface to the level of the frame press. These lift assemblies are mounted on rails with quick release clamps that allow the lift locations to be adjusted in both $\mathrm{x}$ and $\mathrm{y}$ directions, as required to accommodate various size modules. Similarly, the two conveyor belts are mounted with quick release clamps that allow the belt positions to be adjusted in the direction perpendicular to the belt travel direction, as needed for various width modules. These lift and conveyor belt assemblies are similar in design to those used in the trimming system, shown in Figure 7. 
Once the module is raised up on the lift, the two vacuum cups are vented and retracted, leaving the module supported on the five plastic pads, arranged with one near each corner of the module and one at the center. These pads allow the module to slide horizontally as required when the frame members are pressed onto the module edges.

The long frame feeder subassembly, shown in Figure 15, automatically dispenses long frame members from trays and places them on a carriage. The long frame feeder consists of two trays with feed index mechanisms for dispensing frames and two frame pick-and-place mechanisms for transferring frames from the trays to a carriage for sealant dispensing.

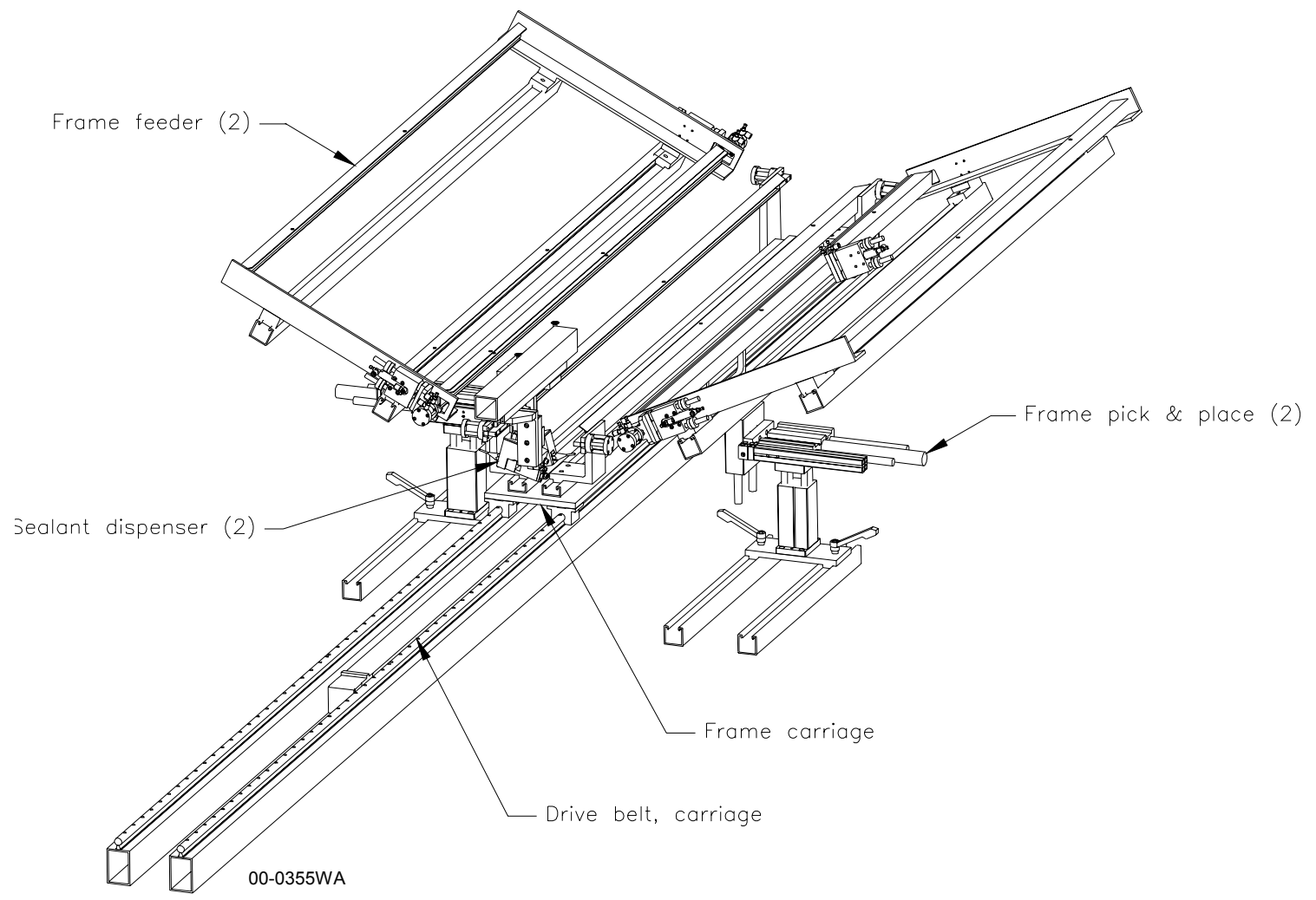

Figure 15 Long frame feeder and carriage assemblies.

Frame feed mechanisms at the bottom of each tray hand off one frame member at a time to a pneumatic pick-and-place mechanism. A side view of the frame tray and feed index mechanism is provided in Figure 16. The index mechanism inserts a pin in the rectangular hole in the bottom-most frame member in the tray to hold the stack in place. Left-handed and right-handed assemblies (i.e. mirror images of each other) were designed to hold both ends of the frame member. When the pick-and-place mechanism acquires the bottom frame member with its vacuum hand, the pins are retracted from the ends of the frame member and the pins are indexed up and inserted into the next frame member on the tray. The pick-and-place mechanism removes the bottom frame from the tray, and the pins index down to reposition the stack for the next hand-off.

A mock-up of the frame tray was fabricated to test the gravity feeding concept for frame members. $147 \mathrm{~cm}\left(58^{\prime \prime}\right)$ long frame members provided by AstroPower were used in this test. These frame members form a $24^{\circ}$ angle with the horizontal when stacked in a nested manner, as shown in Figure 16. 


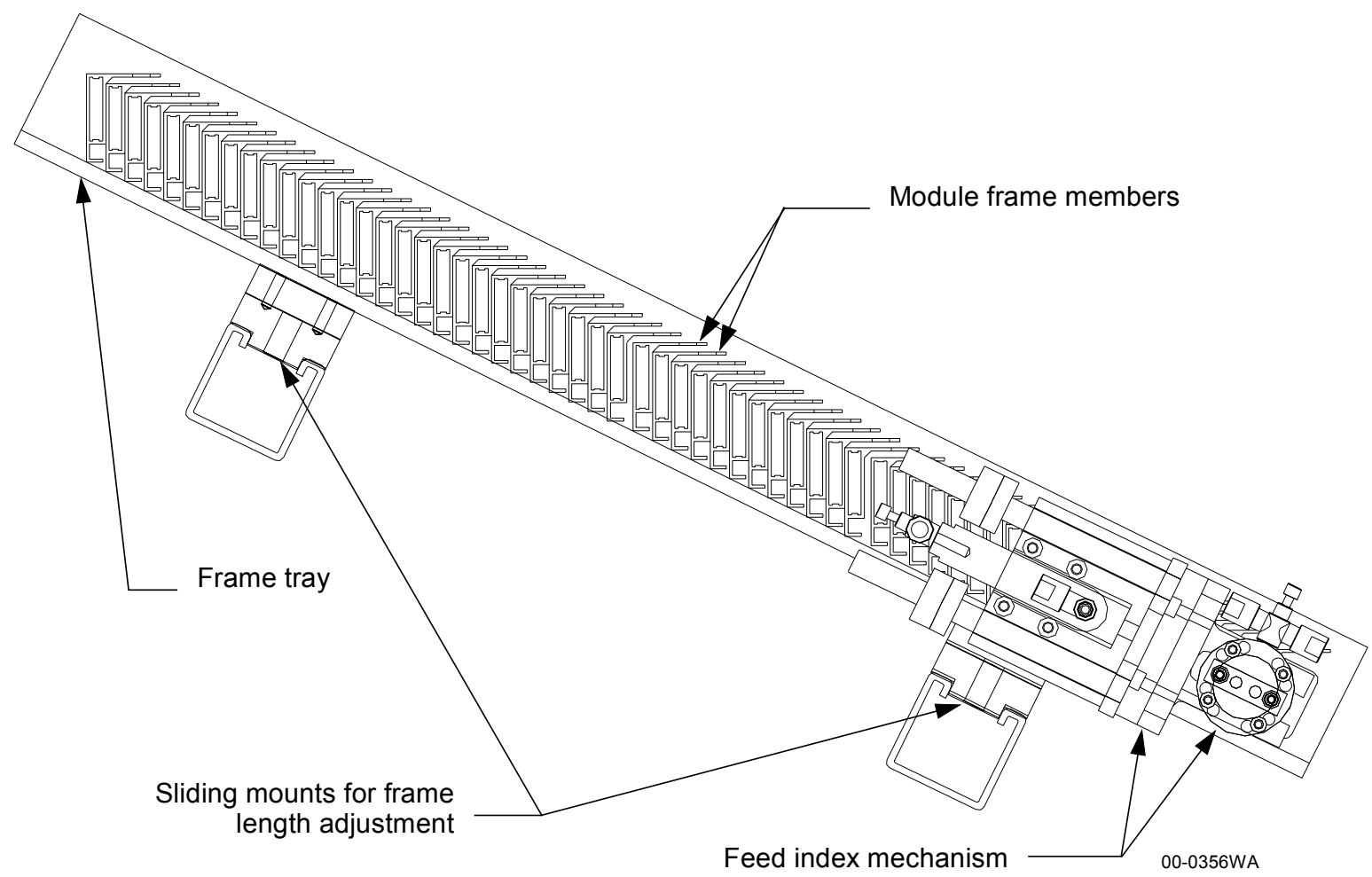

Figure 16 Side view of the frame feeder, shown loaded with 60 frame members.

A stack of frame members was placed on aluminum angles mounted at a $24^{\circ}$ angle to see if the frames will slide down the tray under their own weight as frames are dispensed from the bottom of the stack. The frame members tended to stick to the aluminum angles, so strips of Delrin, ${ }^{4}$ a hard plastic, were attached to the side and bottom surfaces of the angles to reduce friction. With these strips in place, the frames fed smoothly, for both small and large quantities of frames. Thus no pushing mechanism or additional weight is needed. The nested frame stack was found to be stable on the support angles and the frames did not jam inside the tray. As a result, Delrin strips were added to the frame tray assembly to ensure smooth operation.

Two pneumatic pick-and-place assemblies, each with three actuators and a vacuum hand assembly, grip frame members and transport them from the trays to a carriage for sealant dispensing. The pick-and-place mechanism, shown in Figure 17, has one horizontal and two vertical guided cylinders. A plate mounted on channels with quick-release clamps allows the mechanism to be positioned as required for frame members ranging from $91 \mathrm{~cm}$ to $162 \mathrm{~cm}$ (36" to 64") long.

A vacuum hand assembly, shown in Figure 18, was designed for gripping frame members. Four of these subassemblies are used in the framing system, two for the short frame feeder and two for the long frame feeder. Each vacuum cup is independently mounted on spring-loaded linear ball bushings that provide compliance when picking or placing a frame member. Springs are located above and below each bushing to achieve a center-loaded design. When the hand moves up to acquire a frame member, the bushings slide downward for positive sealing of the vacuum cups. Similarly, the bushings slide upward to maintain the vacuum seals when placing a frame member. Independent spring loading of each vacuum cup relaxes the parallelism tolerance for the hand with respect to the assemblies that hold the frames at the pick up and placement locations. 


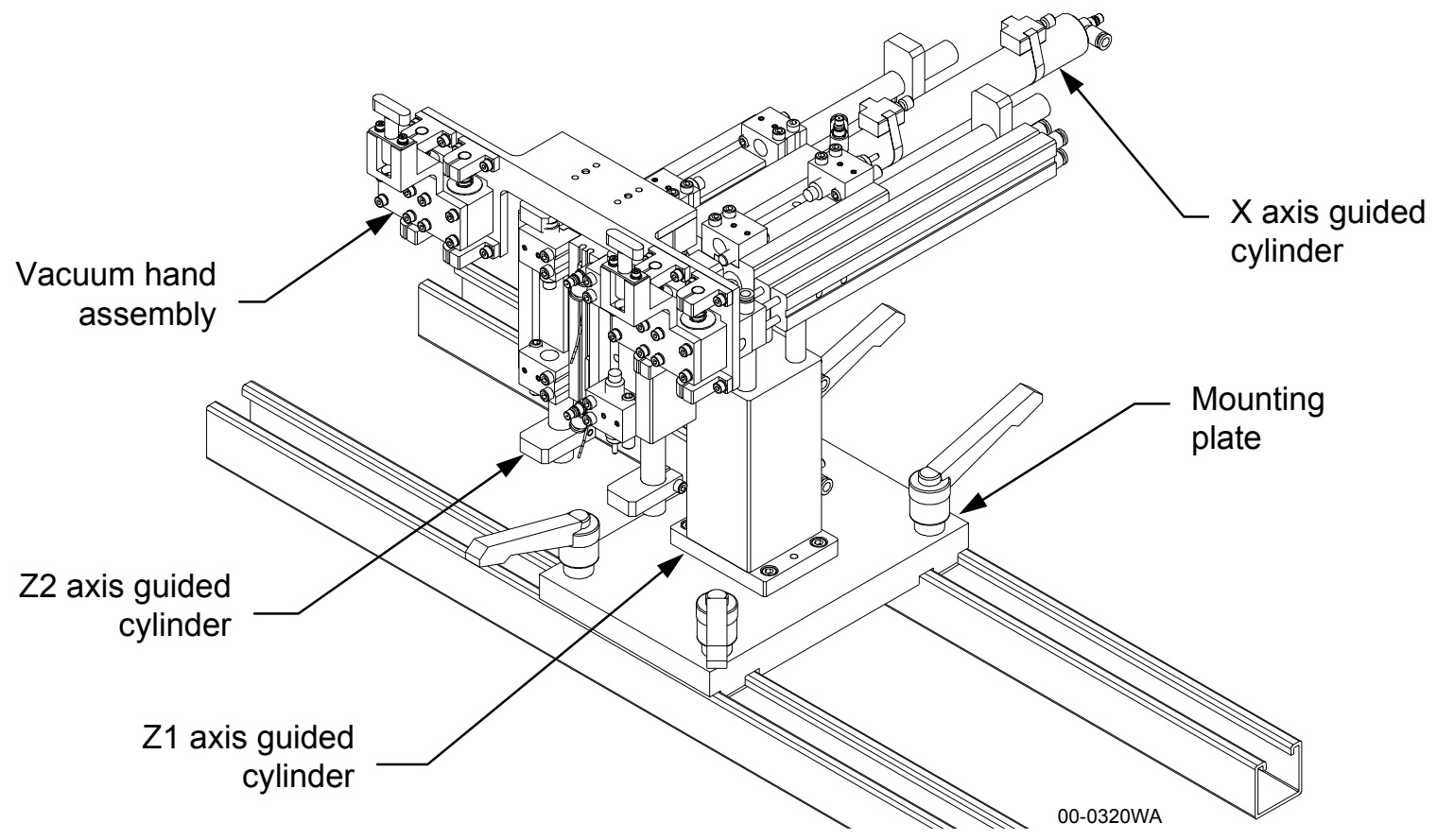

Figure 17 Three-axis pneumatic pick-and-place mechanism for long frame members.

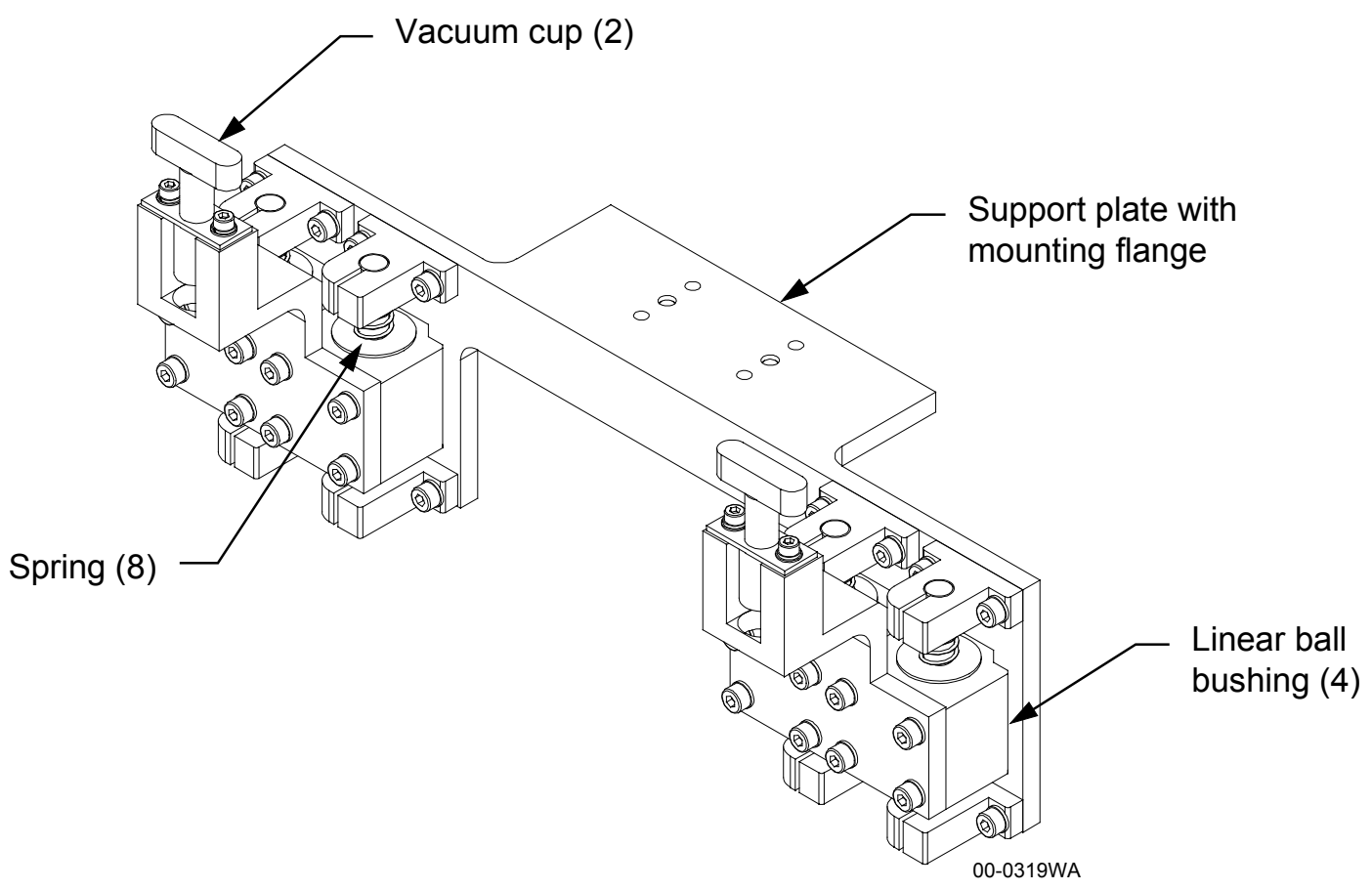

Figure 18 Vacuum hand assembly for long and short frame member pick-and-place assemblies. 
The long frame carriage drives two long frame members at constant velocity past two hot melt sealant nozzles which dispense a bead of sealant into a channel in each frame member. The frame members are then picked up by the robot and transported to the long frame press. The maximum frame member length is $162 \mathrm{~cm}$ (64 inch).

The short frame feeder subassembly, shown in Figure 19, automatically dispenses short frame members from trays, places them in key presses to install corner keys, and places them on a carriage for sealant dispensing. The short frame feeder consists of two trays with feed index mechanisms for dispensing frames, two frame pick-and-place mechanisms for transferring frames from the trays to the key presses and to a carriage, and four key presses with removable key magazines.

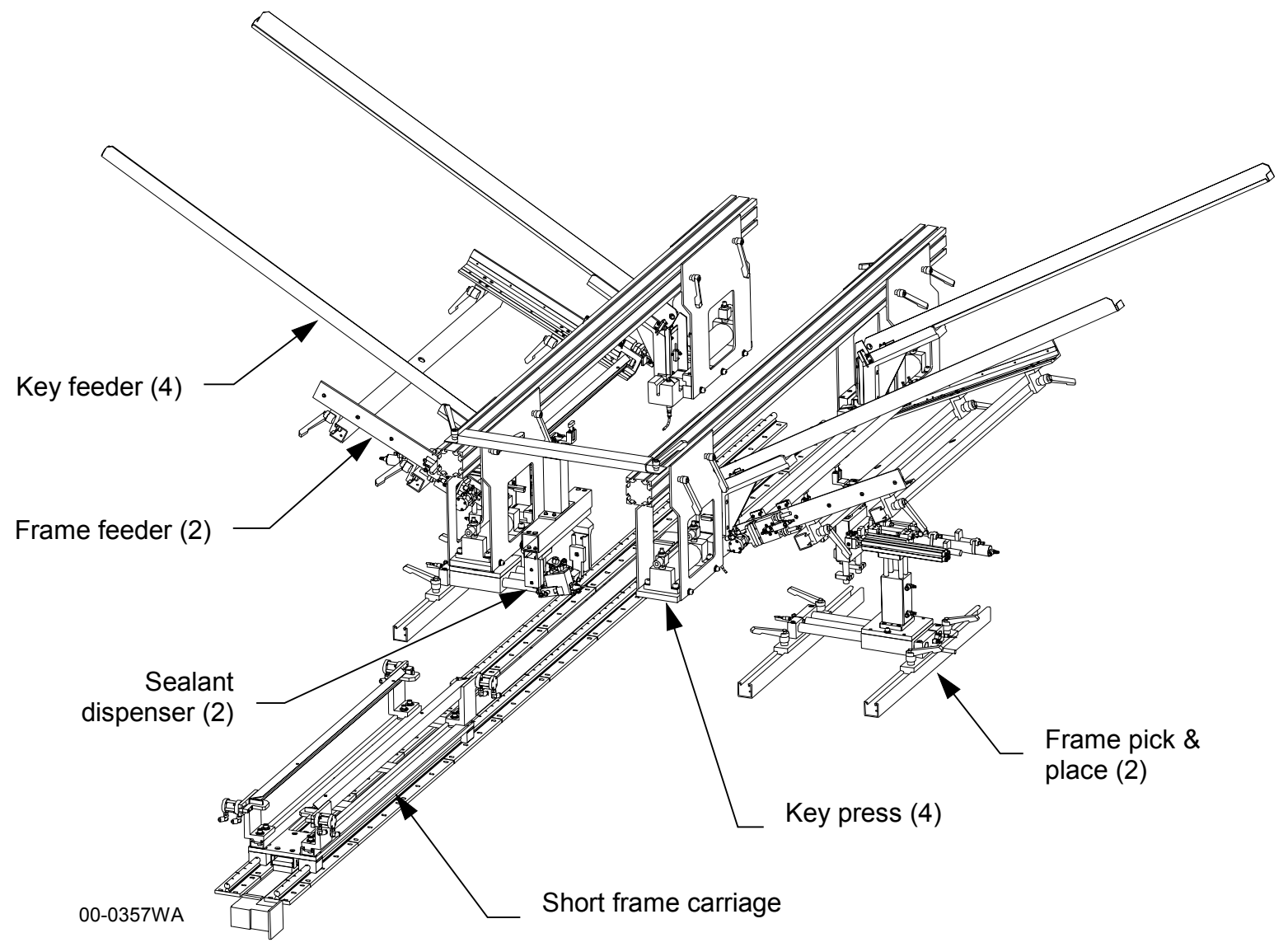

Figure 19 Short frame feeder, key press, and carriage assemblies.

The short frame feeder trays and index mechanisms are similar to those used in the long frame feeder assembly, shown in Figure 16. A four-axis pneumatic pick-and-place mechanism, shown in Figure 20, was designed for dispensing short frame members. The vacuum hand assembly described previously (Figure 18) is mounted on the pick-and-place mechanism for gripping frame members. Two horizontal and two vertical guided cylinders transport frame members from the feed tray to a key press, where corner keys are inserted in both ends of the frame, and from the key press to a carriage for sealant dispensing. Plates mounted on channels with quick-release clamps allows the pick-and-place mechanism to be positioned as needed for frame members ranging from $30 \mathrm{~cm}$ to $102 \mathrm{~cm}$ (12" to 40") long. Two of these subassemblies are used in the short frame feeder assembly. 


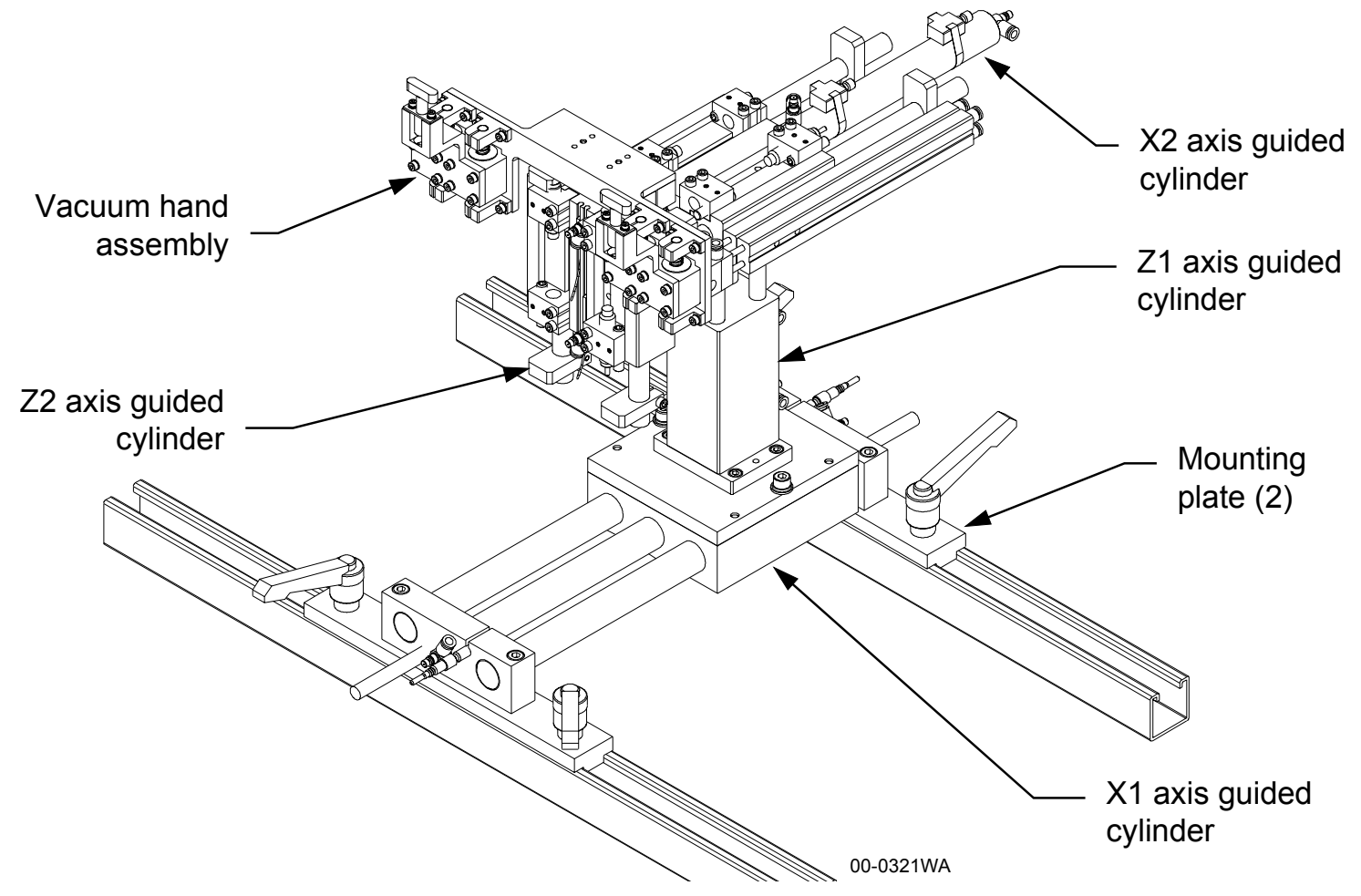

Figure 20 Four-axis pneumatic pick-and-place mechanism for short frame members.

Four corner key feeders and four key presses are provided to dispense L-shaped corner keys and press them into both ends of two short frame members. A gravity-feed concept for dispensing corner keys was developed. The system uses a sloped channel to dispense corner keys directly into each corner key press, as shown in Figure 21. This design greatly simplifies the original key feeder $\operatorname{concept}^{1}$ by eliminating two pneumatic 3-link (x-z- $\theta$ ) pick-and-place mechanisms, two pairs of retracting pins for releasing keys, and two push bars for moving keys to the front of the feeders.

A prototype corner key feeder was fabricated during the design phase to evaluate the gravity feed concept. The feeder was tested with corner keys provided by AstroPower. The tests were successful in proving the basic concept, but also identified some aspects of the design that needed improvement. As a result, the design was modified for easier loading and smoother operation.

Each key feeder includes a key tray that holds up to 60 corner keys, approximately a one hour supply. The tray can be removed from the feeder to simplify key loading. The tray simply slides into the tray support, allowing it to be removed and replaced without tools. A "keys low" optical sensor detects when the last key slides out of the tray, at which point the operator is alerted to fill the tray. The machine will continue to run for several minutes, since there are still several keys in the vertical section of the feeder. This allows time for the operator to refill the tray or insert another pre-filled tray. A "keys empty" sensor detects when the feeder has only two keys left in the vertical section, which stops the key press operation and alerts the operator that the press is out of keys.

Quick-release clamps attach two key press subassemblies to a common extruded aluminum structural support. The clamps tighten nuts in T-slots which run the length of the support, allowing the presses to be positioned anywhere on the support to suit the length of the module frame member. 


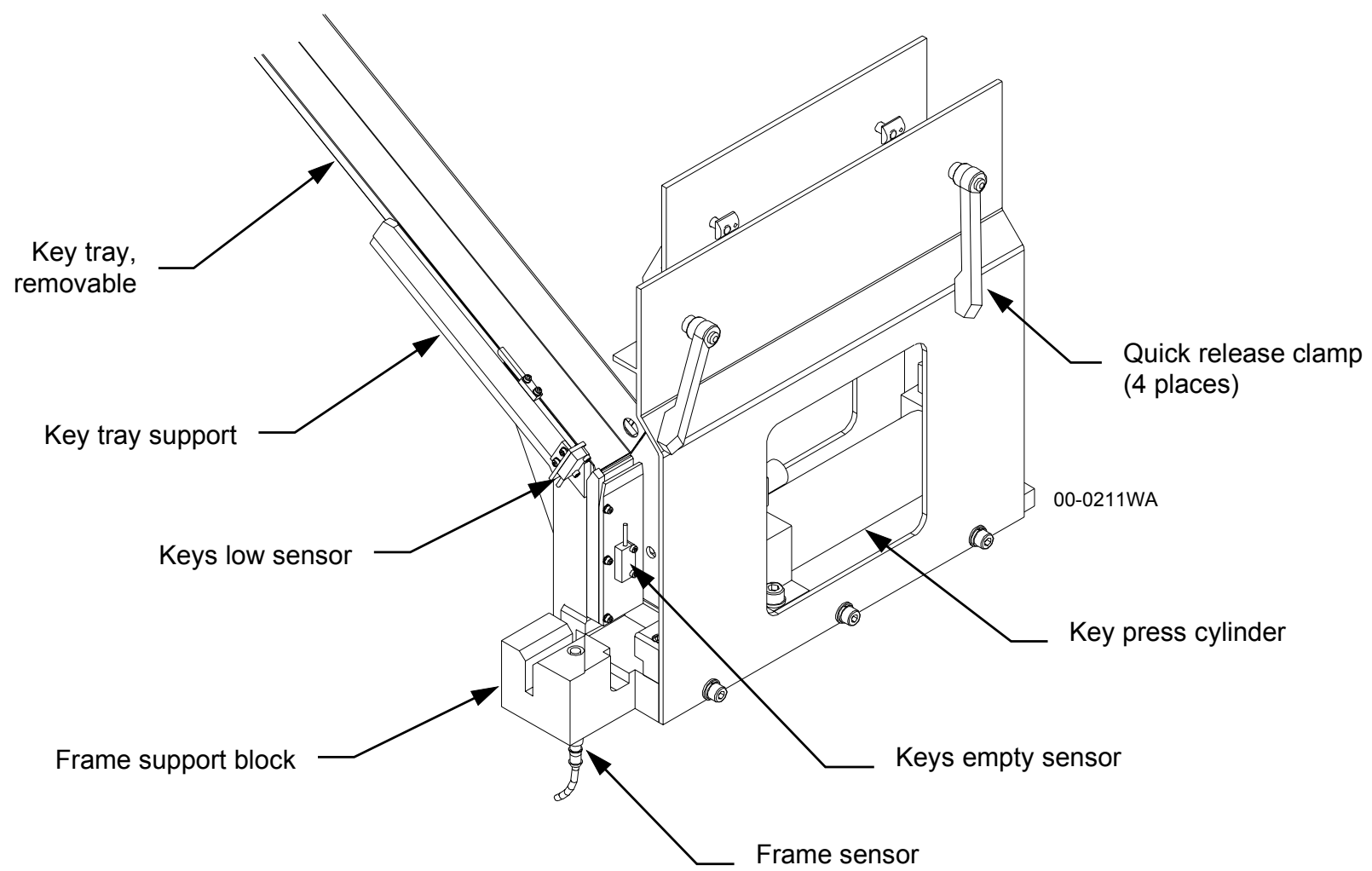

Figure $21 \quad$ Key feeder and press subassembly (right-handed). Only a portion of the key tray is shown. Corner keys and frame member not shown.

Short frame members are transported by the pick-and-place mechanism from a frame feeder and placed on these two key presses, which consist of a left-handed and an opposing right-handed press. Each end of the frame member sits on a support block in the frame press. A proximity sensor in each block detects when the frame is loaded, and signals the two key press cylinders to push the corner keys into both ends of the frame member.

Each press has a block attached to the cylinder rod that presses the key into the frame. The block is designed to hold the stack of keys up while pressing. When the cylinder retracts, gravity causes the stack to fall, automatically loading the next key in the press.

The short frame feeder subassembly includes a carriage and hot melt sealant dispensers that are similar to those used in the long frame feeder. Once the keys are pressed into the ends of the frame members, the pick-and-place mechanisms transport the frames from the key presses to the carriage for sealant dispensing. The carriage assembly, shown in Figure 22, has two pairs of pneumatic clamps to hold two frame members in place. The clamps mount on rails that allow adjustment to match the length of the frame member.

The carriage slides on precision rails with linear ball bushings, powered by a servo motor driven belt. The carriage moves two short frame members at constant velocity past two nozzles which dispense a bead of hot melt sealant into a channel in each frame member. The sealant dispenser assembly is shown in Figure 23. The assembly has adjustments for nozzle angle and nozzle horizontal ( $\mathrm{x}$ and $\mathrm{y}$ ) and vertical positions. 


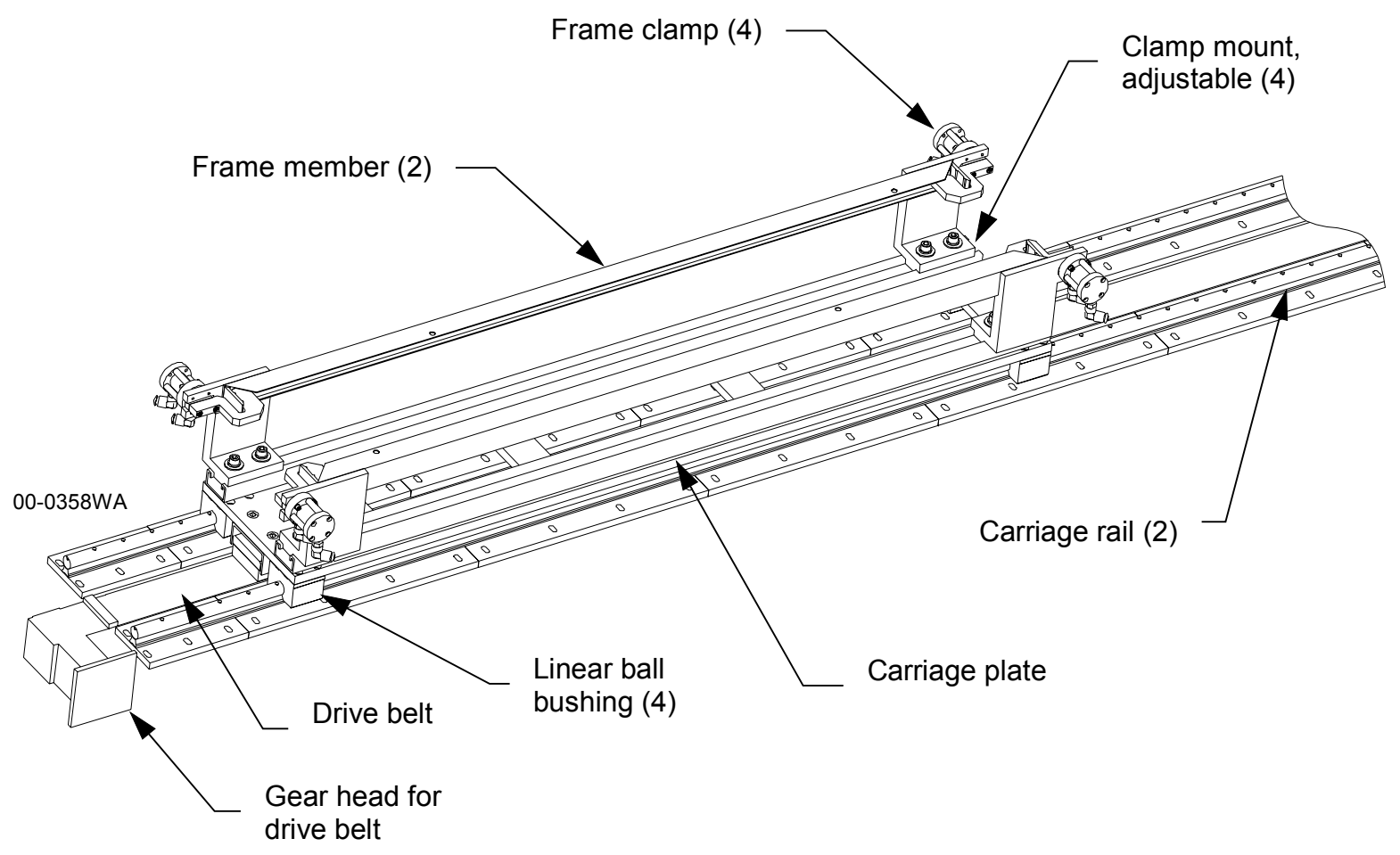

Figure 22 Short frame carriage assembly with two frame members ready for pick-up by the Cartesian robot. Only part of the drive belt and carriage rails is shown.

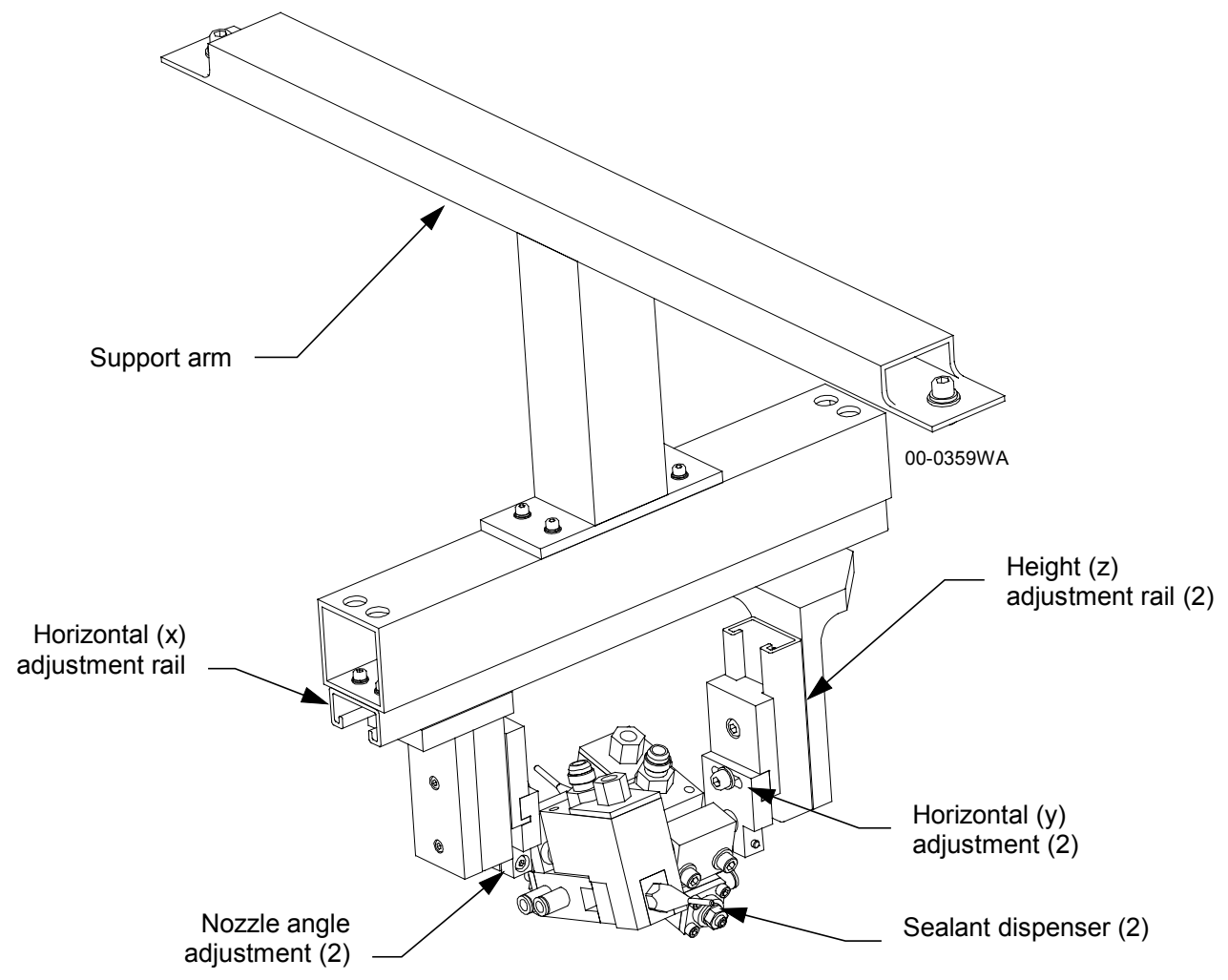

Figure 23 Sealant dispenser and mounting assembly. 
The hot-melt sealant dispensing system includes a heated tank, a sealant pump, two dispensing nozzles with valves, and two heated hoses to deliver sealant from the tank to the nozzles. The tank has a capacity of $22.7 \mathrm{~kg}(50 \mathrm{lbs})$ of sealant. Sealant low and empty sensors were added to the tank. If the sealant level drops to the low sensor position, the operator is alerted to add more sealant to the tank, although the machine continues without interruption. If the sealant level reaches the empty sensor position, the operator is alerted and the machine stops until the sealant tank is refilled.

After sealant is dispensed, the frame members are handed off to the Cartesian robot for transport to the frame press. The four axis (x, y, z, and $\theta$ ) robot (shown in Figure 14) transports an end-effector (Figure 24) with four mechanical grippers that hold two frame members. Frame members are picked up from either the long or short frame carriage and placed in one of four positions in the frame press: the moving and stationary sides of the long frame press, and the moving and stationary sides of the short frame press. The long frame members are rotated $90^{\circ}$ before placement in the press.

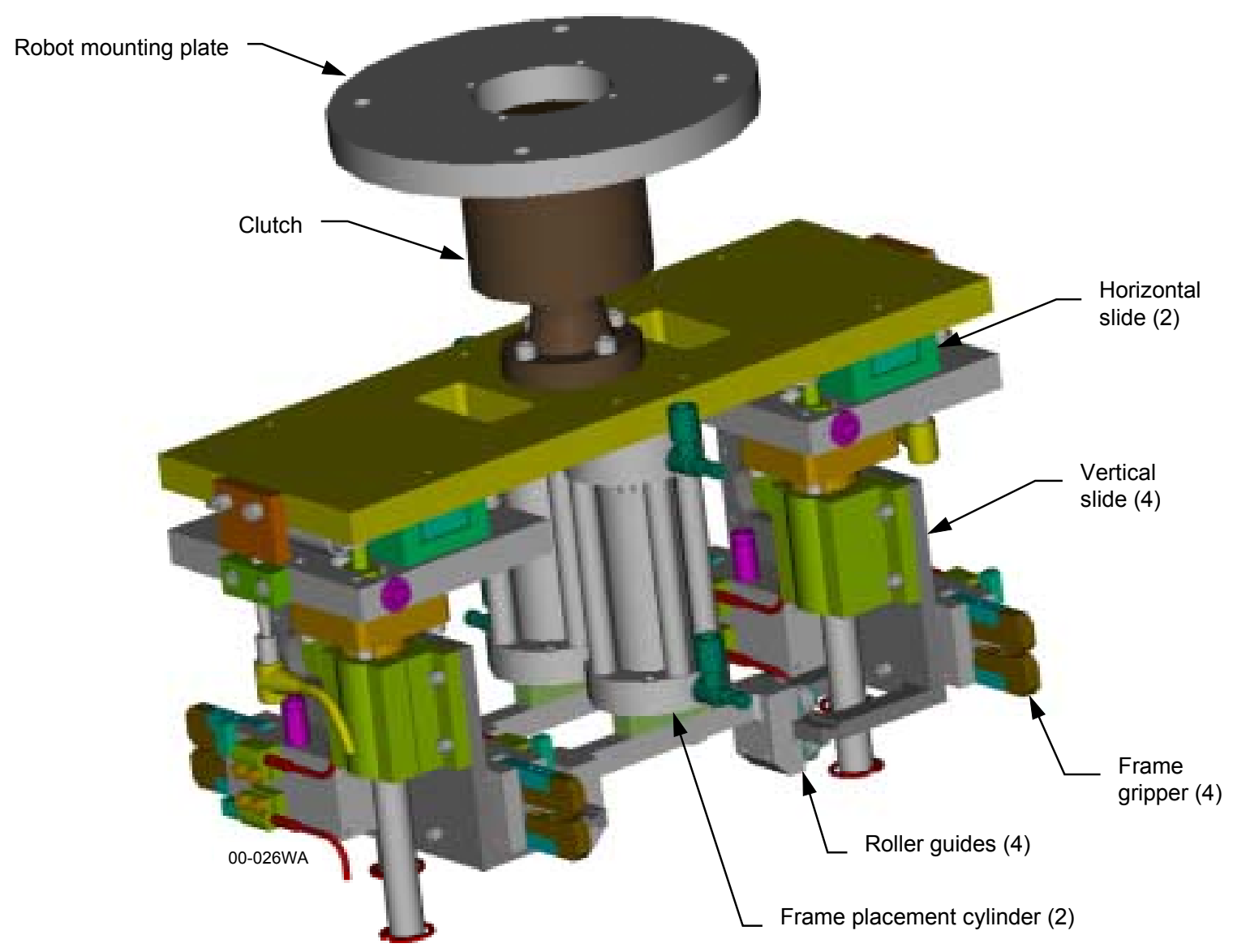

Figure 24 Framer robot end-effector with vertical slides in the up postion and horizontal slides in the center position.

Vertical and horizontal compliance was designed into the robot end-effector to relax tolerance requirements and provide gentle parts handling when placing frames in the press. A mechanical clutch is installed between the end-effector and the robot to protect the robot from damage should a collision occur. 
The end-effector design was modified to enable process improvements identified when framing process flow charts were developed. The vertical linkage was split into two parts, each driven by its own pneumatic cylinder, as shown in Figure 24. This feature enables the robot to place one frame member at a time in the frame press, even if a module is lifted up to the level of the press. This allows the frame press to press two long frame members onto a module before two short frame members are loaded into the press, thereby reducing the time that the hot melt sealant cools in the long frame members before pressing. In addition, the machine cycle time is reduced, since the long frame members can be pressed while the robot retrieves two short frame members from the short frame carriage.

The end-effector has a pair of horizontal slides, which allow the frame sections to be gently placed into position in the frame press. The slides also correct for any small rotational errors introduced by the robot's $\theta$ axis. This horizontal motion was maintained after the two vertical linkages were added to the end-effector by coupling the piston ends of the cylinders to the grippers with rollers that ride in tracks on the gripper brackets.

The frame press design in shown in Figure 25. As the robot places each frame member in the press, air actuated clamps close to hold the frame member in place. Pneumatic cylinders extend to press the frame members up against the edges of the module laminate. Ball bushing slides provide support and prevent the press from racking as it moves. A higher force cylinder is used for the short frame members because it also presses the corner keys attached to the short frame members into the ends of the long frame members.

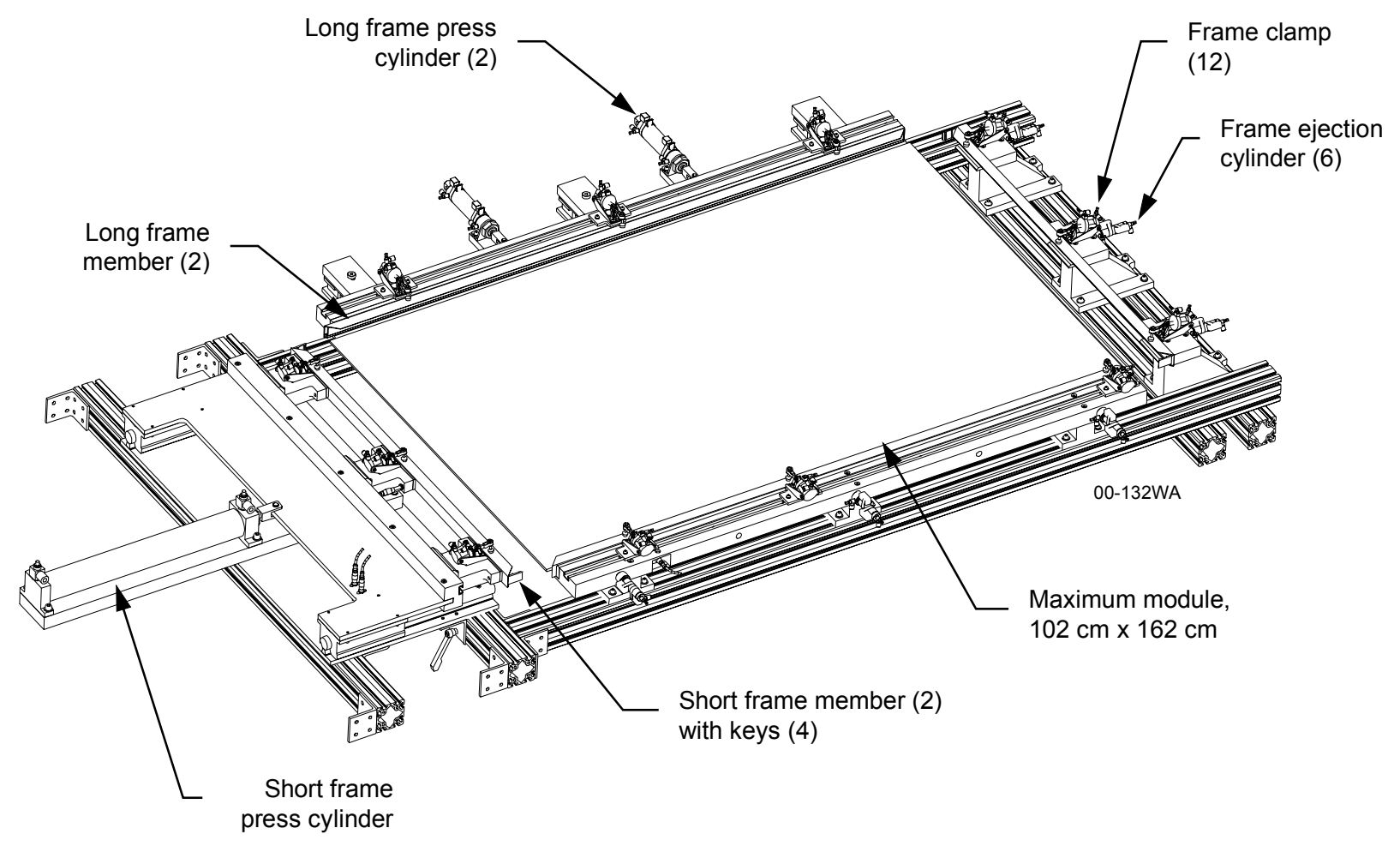

Figure 25 Two-axis frame press subassembly. 
Regardless of module size, the module is always aligned in one corner of the press, near the point where the moving side of the short press meets the stationary side of the long press. The three frame support blocks on both sides of the short frame press are mounted on rails so their positions can adjusted to suit the length of the short frame member. The long frame press has frame member supports that do not need adjustment to accommodate different size frame members, although the positions of the frame clamps can be adjusted. The positions of the support rails for the stationary side of the short press and the moving side of the long press are adjustable, to suit the lengths of the long and short frame members, respectively.

After the long and short frame members are pressed onto the module, the two moving sides of the press retract and ejection pins push the module away from the frame supports at the two stationary sides of the press. The module is now free to be lowered down onto the belt conveyors when the five lift cylinders retract. If the downstream process is ready to receive a module, as indicated by a SMEMA interface signal, the belt conveyors and the output roller conveyor are turned on to transport the module out of the trimming station.

The sealing and framing machine can be grouped into four interdependent subsystems that function in parallel: the short frame subsystem, the long frame subsystem, the Cartesian robot, and the conveyors and frame press subsystem. A top-level flow chart that summarizes these four processes is provided in Figure 26. Detailed flow charts were also developed for each of these four subsystems. These flow charts were used as the basis for specifying sensors and controls and for developing the operating software.

A control system block diagram for the framer is provided in Figure 27. The main controller is an industrial PC running a Microsoft Windows NT operating system. An ethernet connection links the PC with two process controllers for servo motor control and handling digital I/O. Like the trimming system, the framer uses the SMEMA electrical interface ${ }^{3}$ to communicate with upstream and downstream automation.

The main structural frame is enclosed with panels and doors that are electrically interlocked to protect personnel from crushing hazards due to the robot and its end-effector. Transparent acrylic panels are provided in key locations to allow the machine to be observed in operation. Safety guards and devices are also provided to protect personnel from pinch points in the long and short frame loader, key press, and frame carriage areas. Emergency stop buttons are installed around the perimeter of the machine.

A light tower with red, yellow, and green lights indicates the machine status, as described previously in Table 3. The light tower is mounted at the highest point of the machine, on top of the robot frame, for clear visibility in the factory.

Spire's engineering group created 3-D design models for the mechanical assemblies using Autodesk's Mechanical Desktop solid modeling software. From these models, detail drawings were produced for fabricating parts and mechanical assembly drawings were produced to direct the manufacturing group during assembly. A pneumatic schematic was created to document the compressed air and vacuum systems. Electrical wiring drawings and electrical panel layout drawings were created. 


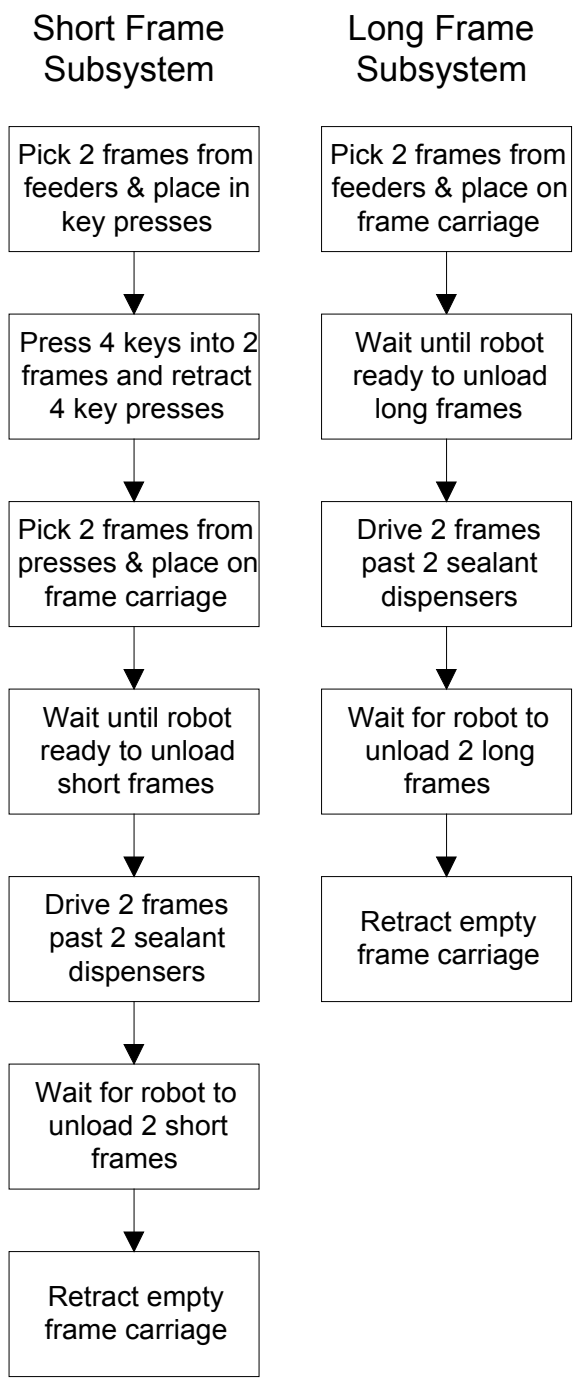

\section{Cartesian \\ Robot}

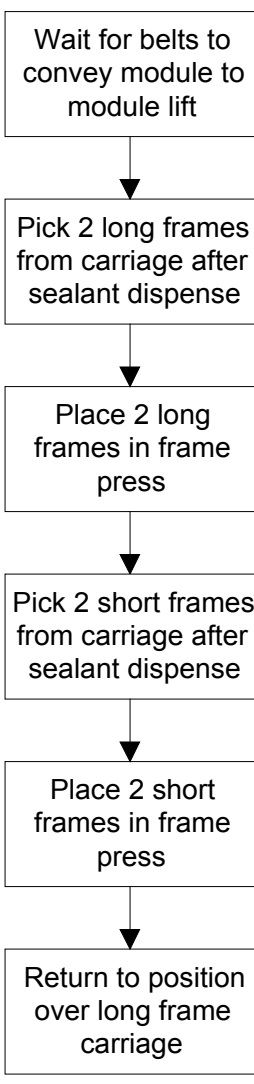

Conveyors \& Frame Press

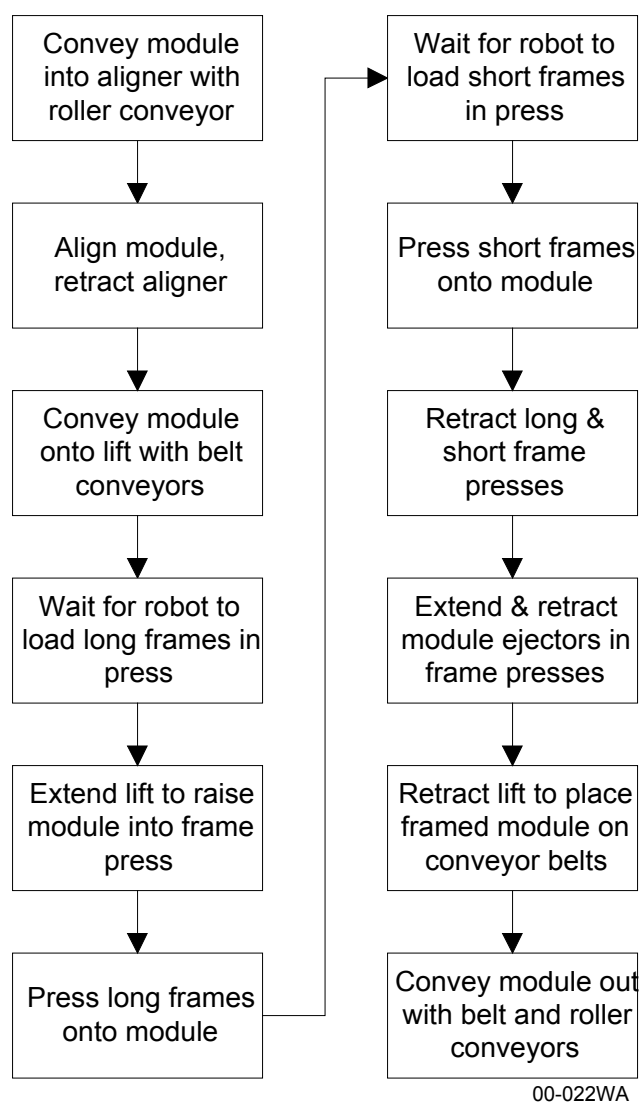

Figure 26 Top-level process flow chart for the module framing system. 


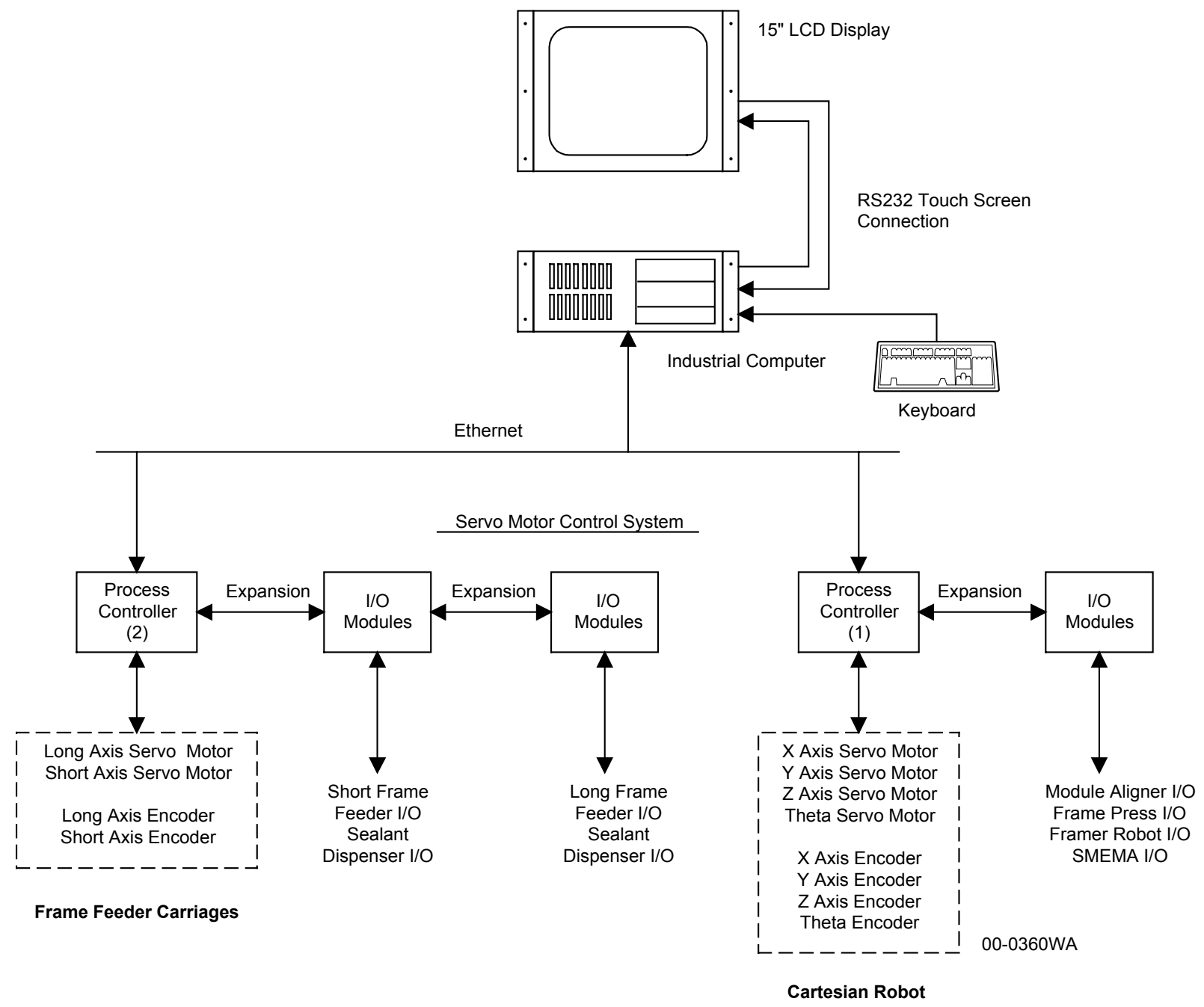

Figure 27 Framer control system block diagram.

The machine control and user interface software is written in Visual Basic. A touch screen provides the main operator interface. The main operating screen is shown in Figure 28. Process parameters are set on a separate screen, shown in Figure 29.

A system parameters screen is provided to set servo motor parameters, such as acceleration, velocity, and home positions, for each of the four Cartesian robot axes and the two frame carriage drive belts. The system parameters are password protected to prevent changes by the operator. Diagnostics screens were also developed to view the status of inputs and outputs. The diagnostics screens allow the outputs to be operated manually (by pressing buttons on the touch screen) to test them for proper operation or to make adjustments for setup or maintenance purposes. 


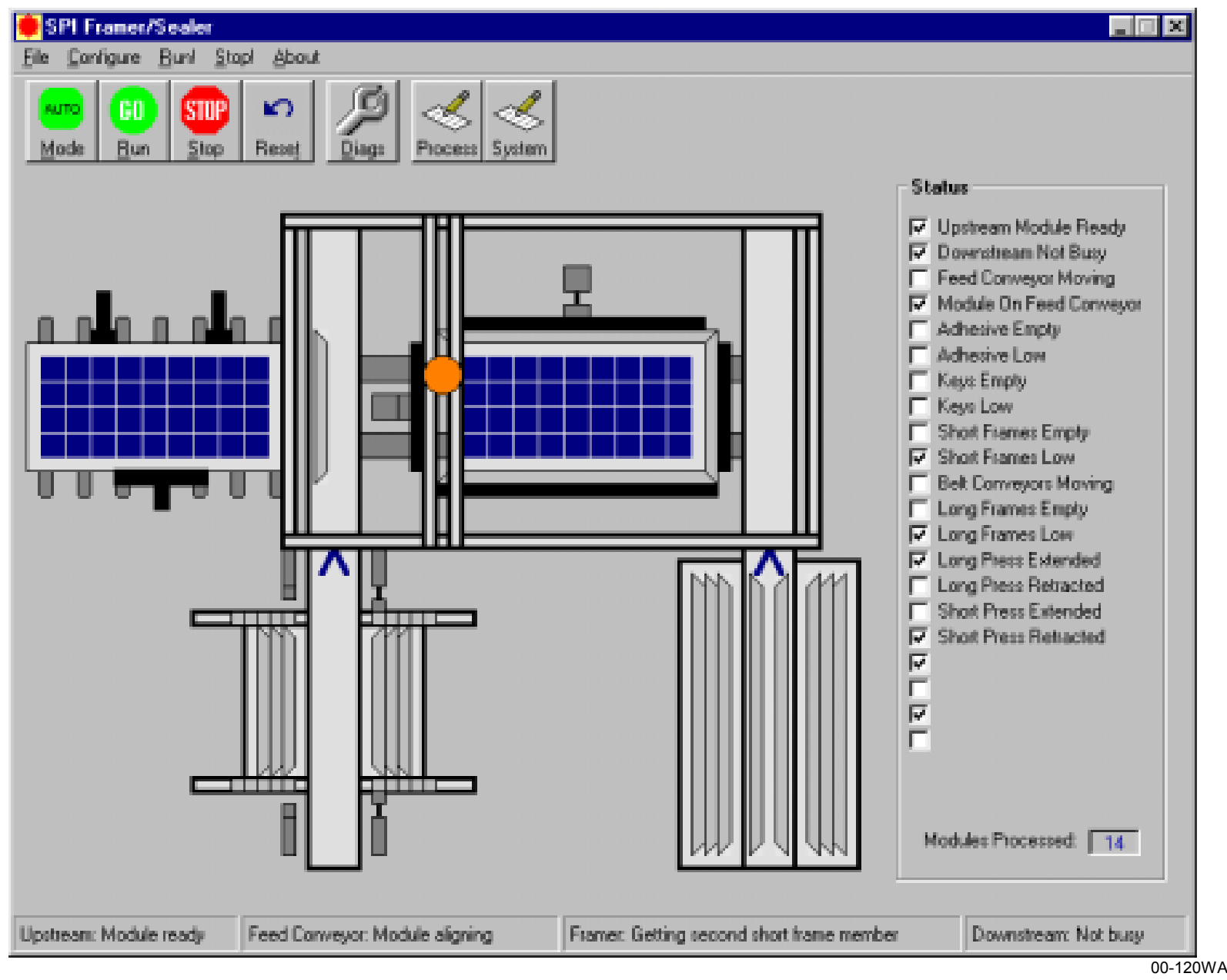

Figure 28 Main operating screen, framer (preliminary).

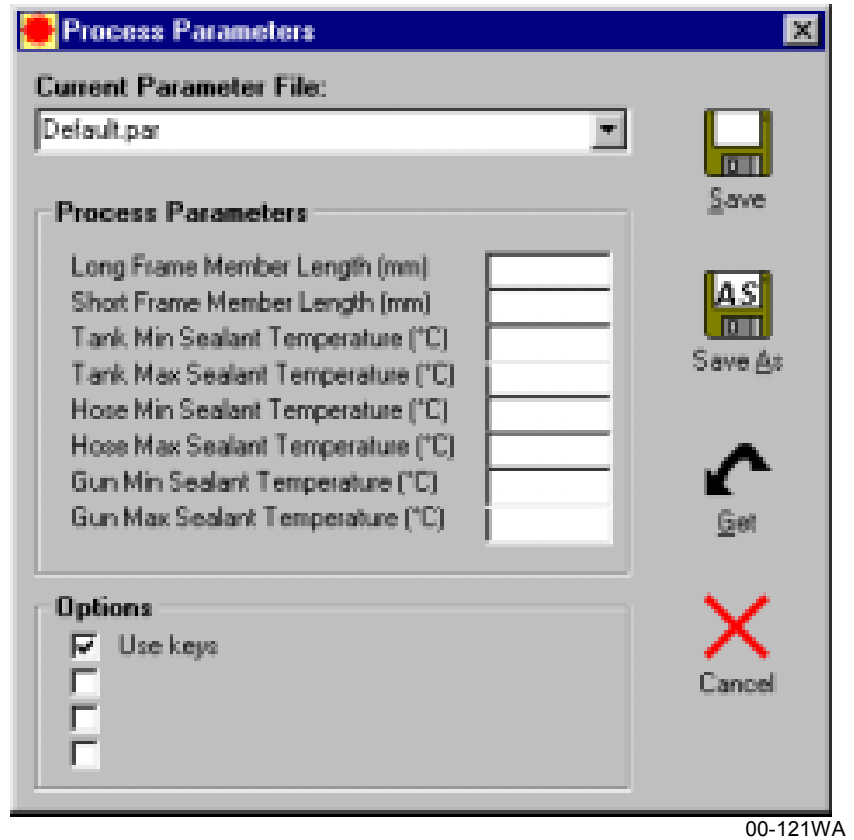

Figure 29 Process parameters screen, framer (preliminary). 


\subsection{Task 6 - Initial Fabrication of Edge Process System}

The automated module edge process system consists of both the trimming system and the edge sealing and framing system. The trimming system was fabricated under Task 6 , while the edge sealing and framing system will be fabricated under Task 7 in Phase 3 of this program.

The module trimming system consists of a number of subassemblies, each of which consists of a number of fabricated and/or purchased parts. Both assembly drawings and detailed part drawings were created during the design process. When the design of each subassembly was completed, a bill of materials was created and the parts were released for fabrication and procurement. Parts were received, inspected, and held in a controlled inventory stockroom until issued to the manufacturing floor for assembly.

Assembly work on the automated trimming system is nearly complete at the end of June, 2000. This work includes the assembly of mechanical, electrical, and pneumatic systems. An overall view of the system is shown in Figure 30. The input conveyor and aligner are shown in Figure 31. The module lift system and the robot end-effector are shown in Figures 32 and 33.

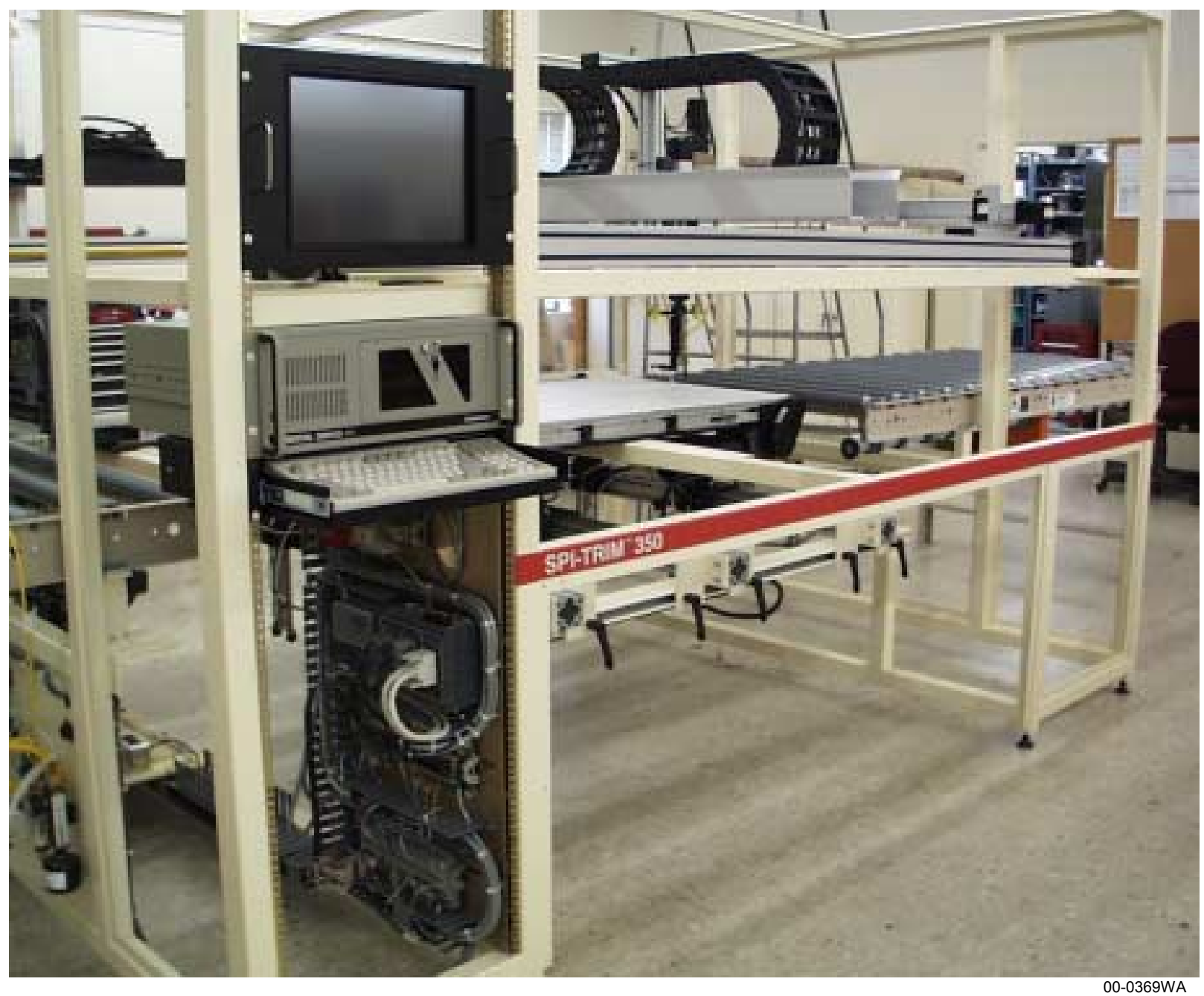

Figure 30 SPI-TRIM 350 automated module trimming system. 


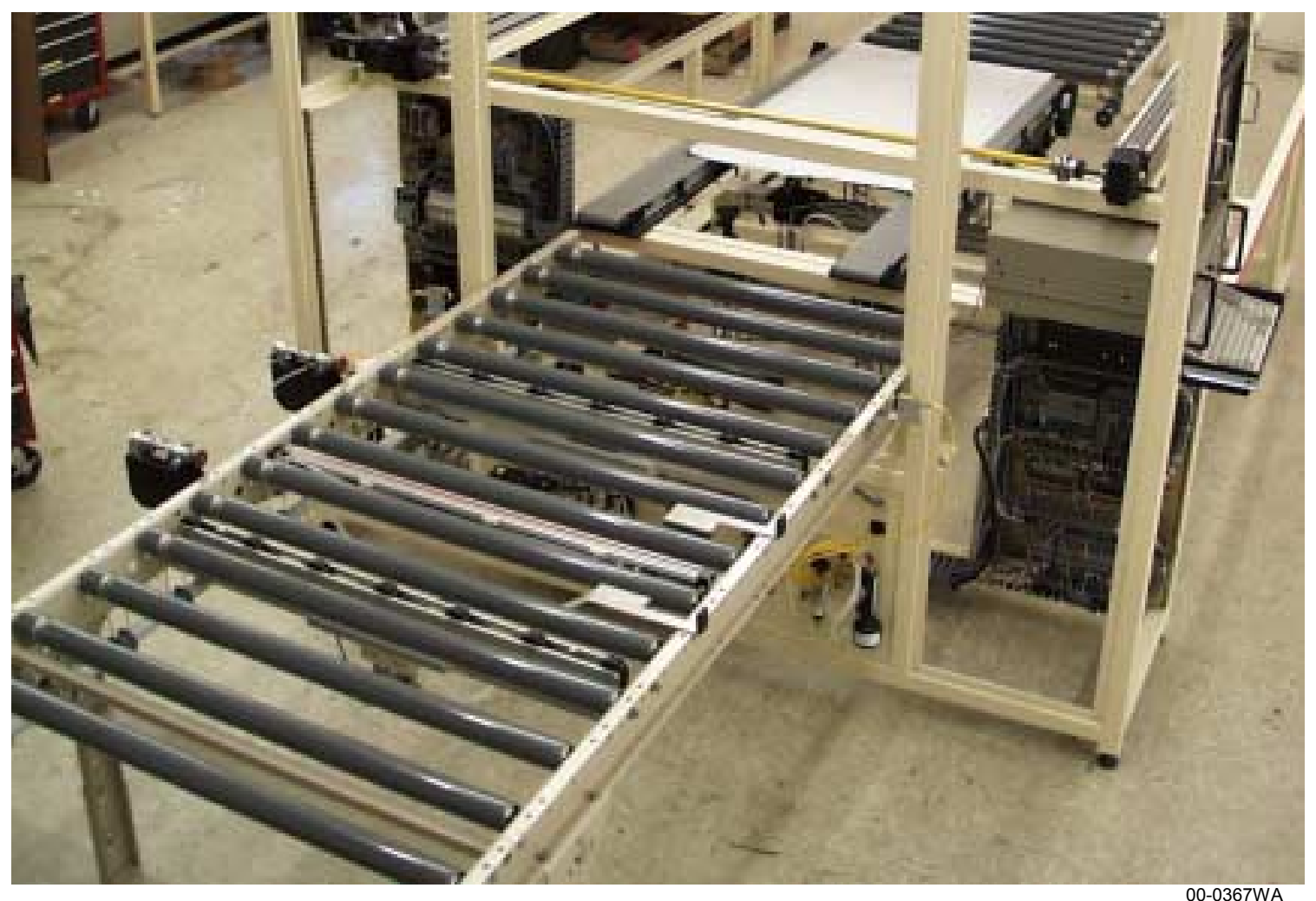

Figure 31 Input conveyor and aligner.

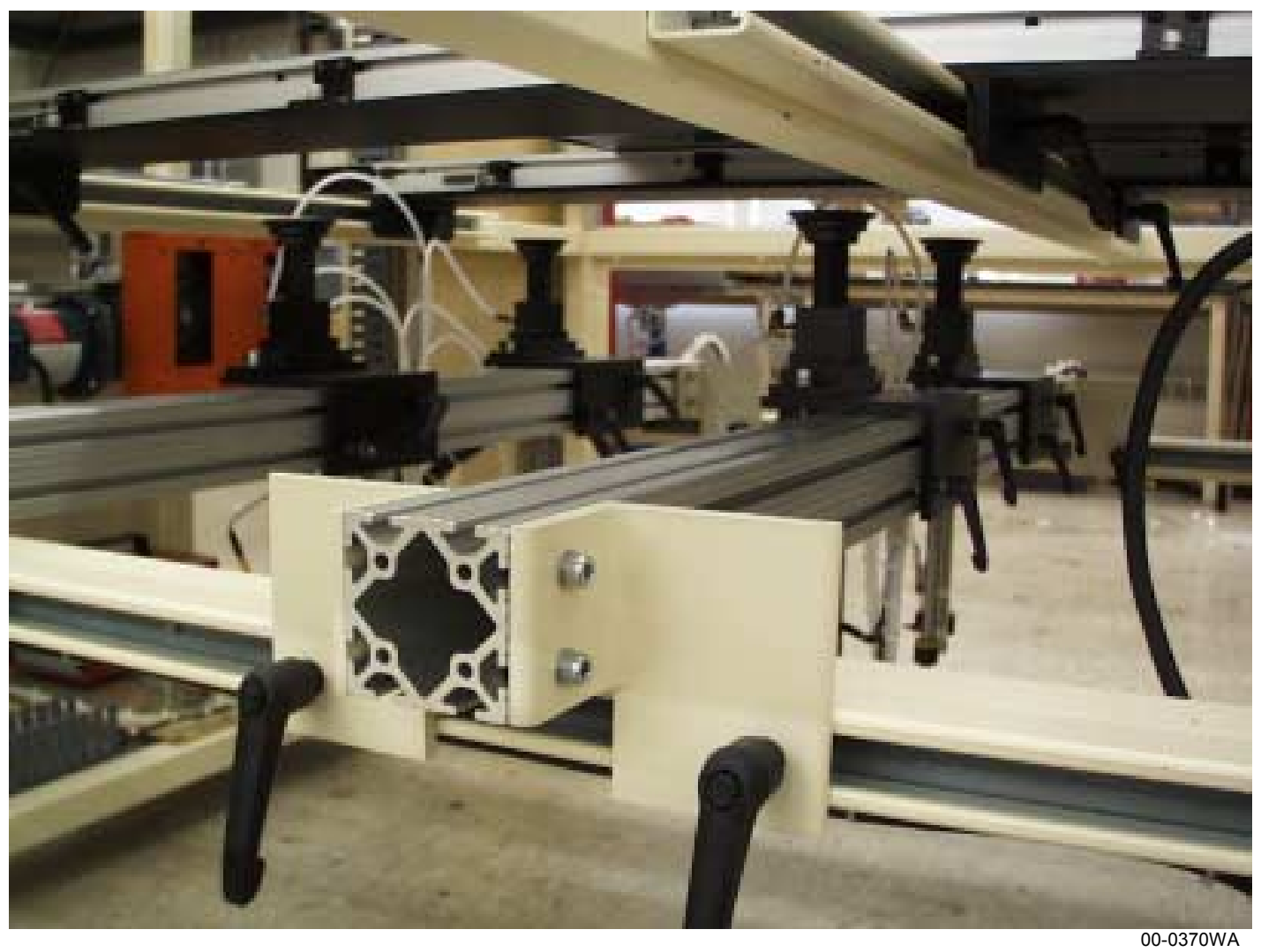

Figure 32 Module lift and belt conveyor systems. 


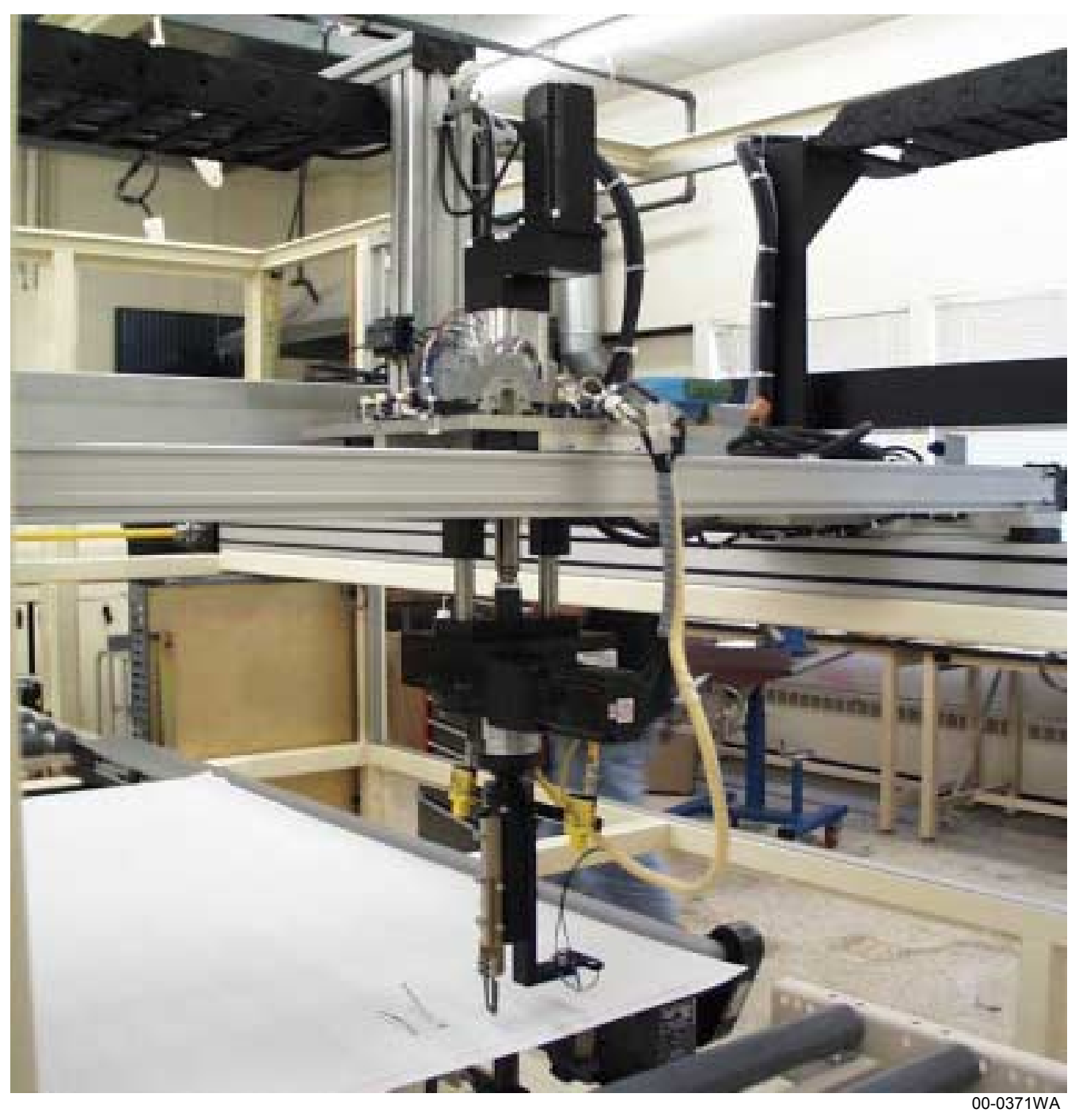

Figure 33 Edge trimmer robot and end-effector.

The trimmer will be powered up and electrical and pneumatic systems will be checked out in July. The automation software running in the PC will be checked out step by step. Adjustments will be made as required to the sensors, limit switches, pneumatic controls, motor controls, and software to achieve proper system operation.

The trimming processes will be evaluated with module laminates from a number of PV module manufacturers. Two crystalline silicon cell manufacturers, AstroPower and Siemens Solar Industries, and an amorphous silicon cell manufacturer, United Solar Systems Corp. (USSC), have indicated a willingness to provide untrimmed laminates for these evaluations. Process parameters will be recorded and cycle times will be measured. 


\section{CONCLUSIONS}

Spire has completed the second phase of a three-phase program for developing new automated post-lamination processes for PV module manufacturing. During this phase, detailed mechanical and electrical designs were completed and software was developed for two prototype production automation systems: a module edge trimming system, designated the SPI-TRIM 350, and a module edge sealing and framing system, designated the SPI-FRAME 350.

These systems can process a broad range of module types, including those made with waferbased and thin-film solar cells. The edge sealing and framing systems described in this report are designed to accommodate modules up to $102 \mathrm{~cm} \mathrm{x} 162 \mathrm{~cm}$, although systems can be produced for larger modules using the same design principles. The goal for process cycle time for both systems is $60 \mathrm{~s}$ per module.

The module edge trimming system was fabricated and will be evaluated with module laminates from several module manufacturers, in July, 2000. The module edge sealing and framing system will be fabricated, tested, and evaluated in Phase 3 of this program. The development of automated processes and a prototype system for junction box installation is also planned for Phase 3.

Automated systems for module buffer storage (SPI-BUFFER 350) and integrated testing (SPIMODULE QA 350) were developed and demonstrated in Phase 1 of this program, which ended in June, 1999. A recently published paper describes the evaluations that were done on the integrated tester, which performs module electrical isolation, ground continuity, and performance (I-V) tests. ${ }^{5}$

All of the automated processes under development in this program are designed to be integrated to create automated production lines. An example of such a line is shown schematically in Figures 2 and 31. The processes are modular and use an industry standard interface protocol ${ }^{3}$ for upstream and downstream handshaking, so they can be arranged in the order desired by the module manufacturer. The use of networked microprocessor controllers (PLCs and PCs) allows real time product tracking and test data acquisition. Bar codes on the backs of modules can be used with scanners to track and assign data to individual modules by serial number.

No commercial off-the-shelf automation was available for these processes prior to this program. Spire has begun marketing these new systems to the PV industry. 


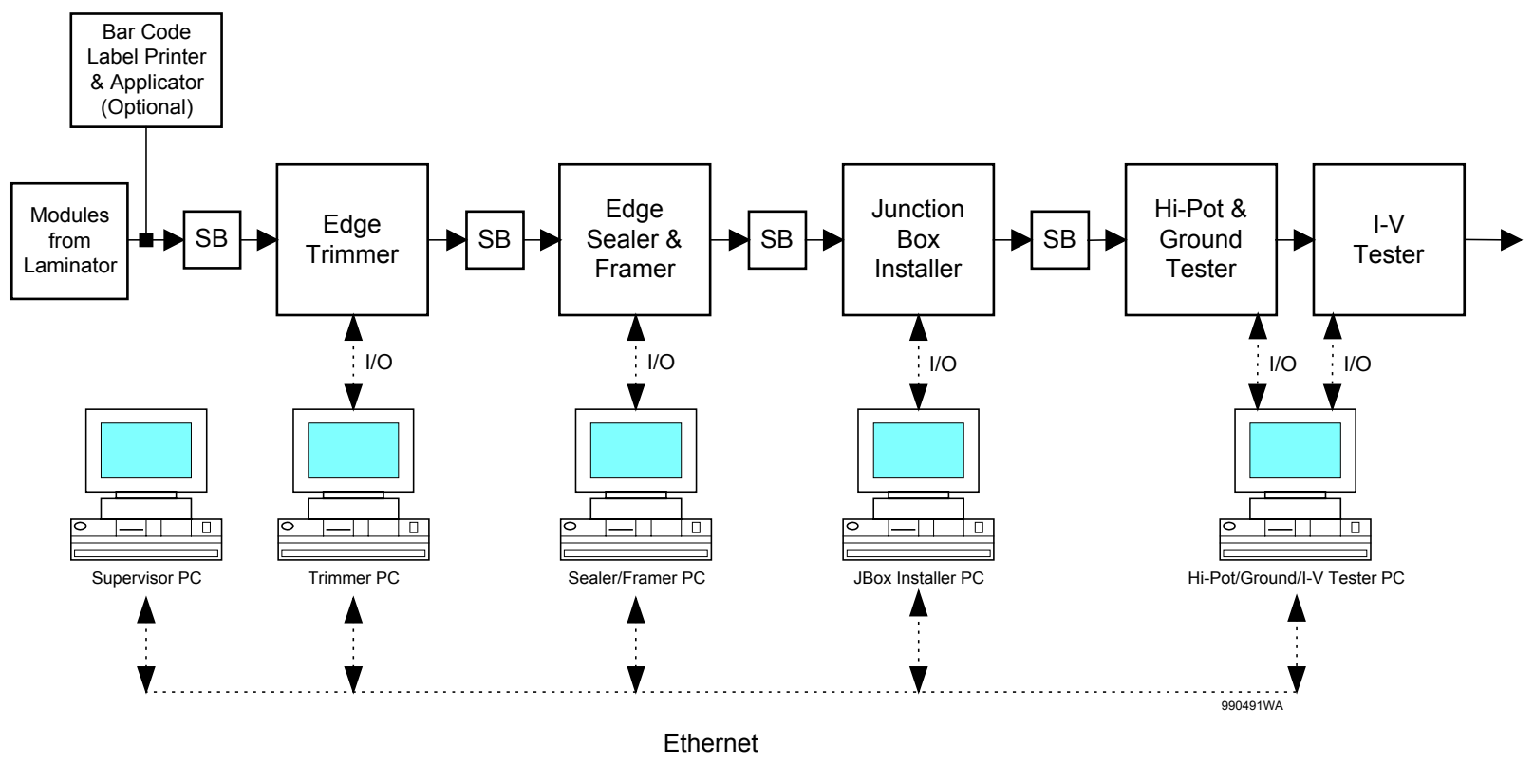

$\mathrm{SB}=$ Storage Buffers

Figure 34 An example of an automated module production line with a network for product tracking and data acquisition. 


\section{REFERENCES}

1. M. J. Nowlan, J. M. Murach, T. W. McCormick, E. R. Lewis, and S. J. Hogan, "Annual Technical Progress Report for Post-Lamination Manufacturing Process Automation for Photovoltaic Modules," Spire doc. ATR-10182-01 (1999).

2. European Standard EN 60204-1, "Safety of Machinery - Electrical Equipment of Machines, Part 1: General Requirements," European Committee for Electrotechnical Standardization (1992).

3. SMEMA Interface Standard 1.2, Surface Mount Equipment Manufacturers Association, Lafayette Hill, PA (1993).

4. Delrin is a registered trademark of DuPont Co. for the acetal homopolymer polyoxymethylene.

5. M. J. Nowlan, J. L. Sutherland, E. R. Lewis, and S. J. Hogan, "Evaluations of an Automated Photovoltaic Module Test System," proc. 16 ${ }^{\text {th }}$ European Photovoltaic Solar Energy Conf., Glasgow, UK, May 1-5, 2000. 


\section{DISTRIBUTION}

2 copies: $\quad$ Dr. Martha I. Symko-Davies, MS \#3214 National Renewable Energy Laboratory 1617 Cole Boulevard

Golden, CO 80401

1 copy: $\quad$ Mr. Michael Quintana, MS \#0752

Sandia National Laboratories

P. O. Box 5800

Albuquerque, NM 87185-0752

1 copy: $\quad$ Mr. Steve Rummel, MS \#3411

National Renewable Energy Laboratory

1617 Cole Boulevard

Golden, CO 80401

1 copy: $\quad$ Ms. Victoria Lewis, MS \#2713

National Renewable Energy Laboratory

1617 Cole Boulevard

Golden, CO 80401 


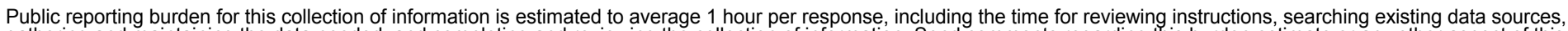

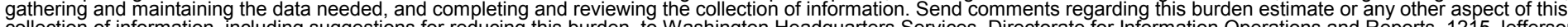

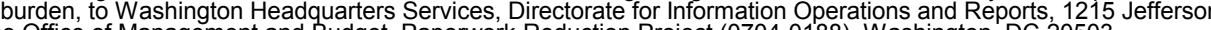
Davis Highway, Suite 1204, Arlington, VA 22202-4302, and to the Office of Management and Budget, Paperwork Reduction Project (0704-0188), Washington, DC 20503.

\begin{tabular}{|l|l|l} 
1. AGENCY USE ONLY (Leave blank) & $\begin{array}{l}\text { 2. REPORT DATE } \\
\text { September 2000 }\end{array}$ & $\begin{array}{l}\text { 3. REPORT TYPE AND DATES COVERED } \\
\text { Annual Technical Progress Report, } \\
15 \text { June 1999-14 July 2000 }\end{array}$ \\
\hline
\end{tabular}

4. TITLE AND SUBTITLE

Post-Lamination Manufacturing Process Automation for Photovoltaic Modules;

Annual Technical Progress Report, 15 June 1999-14 July 2000

6. AUTHOR(S)

M.J. Nowlan, J.M. Murach, S.F. Sutherland, E.R. Lewis, and S.J. Hogan

7. PERFORMING ORGANIZATION NAME(S) AND ADDRESS(ES)

Spire Corporation

5. FUNDING NUMBERS

C: ZAX-8-17647-04

TA: PV006101

One Patriots Park

Bedford, MA 01730-2396

9. SPONSORING/MONITORING AGENCY NAME(S) AND ADDRESS(ES)

National Renewable Energy Laboratory

1617 Cole Blvd.

Golden, CO 80401-3393

8. PERFORMING ORGANIZATION REPORT NUMBER

11. SUPPLEMENTARY NOTES

NREL Technical Monitor: M. Symko-Davies

12a. DISTRIBUTION/AVAILABILITY STATEMENT

12b. DISTRIBUTION CODE

National Technical Information Service

U.S. Department of Commerce

5285 Port Royal Road

Springfield, VA 22161

13. ABSTRACT (Maximum 200 words): Spire is addressing the PVMaT project goals of photovoltaic (PV) module cost reduction and improved module manufacturing process technology. New cost-effective automation processes are being developed for post-lamination PV module assembly, where post-lamination is defined as the processes after the solar cells are encapsulated. These processes apply to both crystalline and thin-film solar cell modules. Four main process areas are being addressed:

- Module buffer storage and handling between steps

- Module edge trimming, edge sealing, and framing

- Junction-box installation

- Testing for module performance, electrical isolation, and ground-path continuity.

14. SUBJECT TERMS photovoltaics; post-lamination module manufacturing; PVMaT; edge processes; trimming; sealant; framing; thermocouple connector; optical sensor; initial fabrication; process parameters

17. SECURITY CLASSIFICATION OF REPORT Unclassified
18. SECURITY CLASSIFICATION OF THIS PAGE Unclassified
19. SECURITY CLASSIFICATION OF ABSTRACT Unclassified
15. NUMBER OF PAGES

16. PRICE CODE

20. LIMITATION OF ABSTRACT

UL 